\title{
Are there jumps in evidence accumulation, and what, if anything, do they reflect psychologically? An analysis of Lévy-Flights models of decision-making
}

\author{
Amir Hosein Hadian Rasanan* \\ Institute for Cognitive and Brain Sciences, Shahid Beheshti University, Tehran, Iran \\ Faculty of Psychology, University of Basel, Basel, Switzerland \\ Jamal Amani Rad ${ }^{\dagger}$ \\ Department of Cognitive Modeling, Institute for Cognitive and Brain Sciences, \\ Shahid Beheshti University, Tehran, Iran \\ David K. Sewell $\ddagger$ \\ School of Psychology, The University of Queensland, St Lucia, QLD 4072, Australia
}

*Email: amir.h.hadian@gmail.com; amirhosein.hadianrasanan@unibas.ch

†Email: j_amanirad@sbu.ac.ir; j.amanirad@gmail.com

${ }_{\ddagger}^{\ddagger}$ Email: d.sewell@uq.edu.au (Corresponding author) 


\begin{abstract}
According to existing theories of simple decision-making, decisions are initiated by continuously sampling and accumulating perceptual evidence until a threshold value has been reached. Many models, such as the diffusion decision model, assume a noisy accumulation process, described mathematically as a stochastic Wiener process with Gaussian distributed noise. Recently, an alternative account of decision-making has been proposed in the Lévy Flights (LF) model, in which accumulation noise is characterized by a heavy-tailed power-law distribution, controlled by a parameter, $\alpha$. The LF model produces sudden large "jumps" in evidence accumulation that are not produced by the standard Wiener diffusion model, which some have argued provide better fits to data. It remains unclear, however, whether jumps in evidence accumulation have any real psychological meaning. Here, we investigate the conjecture by Voss et al. (2019) that jumps might reflect sudden shifts in the source of evidence people rely on to make decisions. We reason that if jumps are psychologically real, we should observe systematic reductions in jumps as people become more practiced with a task (i.e., as people converge on a stable decision strategy with experience). We fitted four versions of the LF model to behavioral data from a study by Evans and Brown (2017), using a five-layer deep inference neural network for parameter estimation. The analysis revealed systematic reductions in jumps as a function of practice, such that the LF model more closely approximated the standard Wiener model over time. This trend could not be attributed to other sources of parameter variability, speaking against the possibility of trade-offs with other model parameters. Our analysis suggests that jumps in the LF model might be capturing strategy instability exhibited by relatively inexperienced observers early on in task performance. We conclude that further investigation of a potential psychological interpretation of jumps in evidence accumulation is warranted.
\end{abstract}

Keywords: Decision making, Evidence accumulation, Lévy Flights, Jump, Speed-accuracy tradeoff, Heavy-tailed distribution.

\title{
Introduction
}

Theories of decision-making are fundamental to our understanding of psychology, neuroscience, and neuroeconomics, and have been developed and systematically tested over the last 60 years (Evans and Wagenmakers, 2020; Ratcliff et al., 2016; Sewell and Smith, 2016). The dominant view that has emerged over this time is that simple decisions can be conceptualized as a noisy evidence accumulation process, and mathematically represented by the class of sequential sampling models (SSMs; Ratcliff and Smith, 2004; Stone, 1960). In these models, stimulus information is continuously sampled, with each sample contributing a quantity of evidence favoring one or more response alternatives. The evidence from successive samples is summed, or accumulated through time until a criterion amount of evidence for one response alternative is accrued, initiating a behavioral response for that alternative. The success of this theoretical framework is reflected in the 
breadth of domains the models have been applied to, such as evaluating the optimality of decision policies (Bogacz et al., 2006; Drugowitsch et al., 2012; Evans et al., 2020; Evans and Brown, 2017; Evans et al., 2018; Starns and Ratcliff, 2012), stop signal paradigms (Matzke et al., 2013, 2017a,b), Go/No-Go paradigms (Gomez et al., 2007; Ratcliff et al., 2018), multi-attribute and many-alternatives choice (Mallahi-Karai and Diederich, 2019, 2021; Kvam, 2019; Roe et al., 2001; Trueblood et al., 2014; Usher and McClelland, 2004), learning strategies (Fontanesi et al., 2019; Miletić et al., 2021; Pedersen et al., 2017; Sewell et al., 2019; Sewell and Stallman, 2020), attentional choice (Krajbich et al., 2010, 2012; Gluth et al., 2020), continuous responses (Ratcliff, 2018; Smith, 2016), neural processes (Gold and Shadlen, 2007), and so on.

The most popular and well-studied model of simple decision-making is the diffusion decision model (DDM) of Ratcliff (Ratcliff, 1978; Ratcliff and Mckoon, 2008; Ratcliff and Rouder, 1998a). The DDM represents evidence as a single signed value, $X(t)$, that is accumulated from a starting point, $z$, toward one of two absorbing boundaries, located at zero and $a$. Psychologically, the boundary separation parameter, $a$, characterizes response caution, with more widely separated boundaries reflecting more cautious responding (i.e., a greater quantity of evidence is required to trigger a response). The start-point parameter, $z$, is interpreted psychologically as characterizing response bias, and varies uniformly with mean $z$ and range $s_{z}$. An unbiased decision-maker will begin evidence accumulation from $z=\frac{a}{2}$. For a biased decision-maker, the accumulation process will begin closer to the boundary favored by the bias. The rate of evidence accumulation, the drift rate of the diffusion process, has a mean $v$ and standard deviation $\eta$ across trials. Psychologically, the drift rate is determined by the quality of the stimulus. The sign of the drift rate determines which response alternative evidence tends to accumulate towards. Drift rates with absolute values that deviate further from zero correspond to stimuli with higher-quality information that more readily discriminates between response alternatives. Mathematically, evidence accumulation in the DDM can be described as,

$$
X(t+\Delta t)=X(t)+v \cdot \Delta t+\mathcal{N}(0,1) \cdot \sqrt{\Delta t}
$$

where $\mathcal{N}$ denotes a standard normal distribution and $\Delta t$ represents the time step. The accumulation dynamics of Equation 1 determine the decision time, but behavioral response times also consist of the time required to encode the stimulus and execute a response. In the DDM, these components of non-decision time are described by the parameter, $t_{0}$ (also written as $T_{e r}$ ). Non-decision time is assumed to vary uniformly with mean $t_{0}$ and range st. Components of the DDM (i.e., without variability parameters) are illustrated in Figure 1. 
Diffusion Decision Model

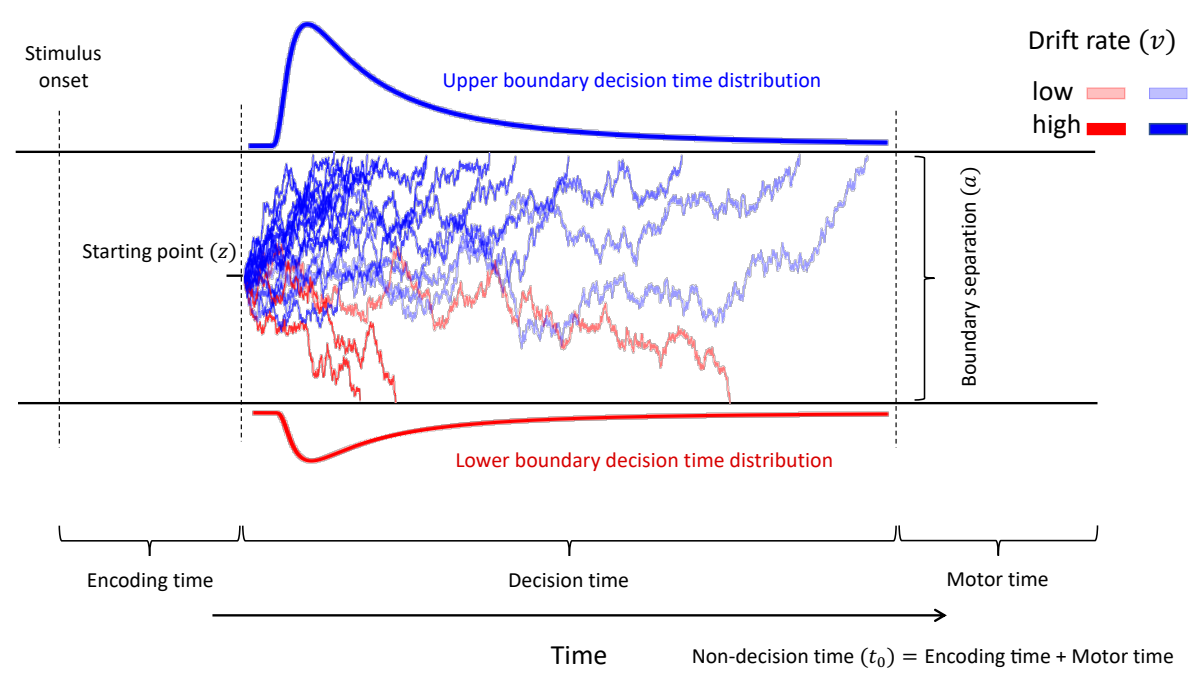

Figure 1. A schematic view of the diffusion decision model. The jagged lines depict evidence accumulation trajectories driven by different stimuli across different trials. The darker lines correspond to higher-quality stimuli that produce drift rates that deviate further from zero. The blue and red trajectories were produced by different stimuli (e.g., rightward vs. leftward motion), which drive the accumulation process toward different absorbing boundaries. The reader is referred to the online version of this article for a colored version of the figure.

A number of alternatives to the DDM have been developed. These include "reduced" forms of the standard DDM, incorporating simplifying assumptions that remove between-trial parameter variability (Wagenmakers et al., 2007), as well as models that make different assumptions about how evidence is represented in the model. For example, multi-accumulator models (e.g., (Brown and Heathcote, 2005, 2008; Hawkins and Heathcote, 2021; Smith and Vickers, 1988; Tillman et al., 2020; Usher and McClelland, 2001)), represent separate absolute evidence totals for each response alternatives that race against each other, rather than a single signed relative evidence total. More recently, Voss and colleagues (Voss et al., 2019; Wieschen et al., 2020) have proposed the Lévy Flights (LF) model as another alternative, which includes the DDM as a special case. The LF model differs from the DDM in assuming that there are random "jumps" in evidence accumulation that do not conform to the standard Gaussian noise process described in Equation 1. To allow for these large sudden changes in evidence accumulation, the noise in the accumulation process is instead characterized by a heavy-tailed $\alpha$-stable distribution with long-tailed, power-law asymptote $\lambda(x) \sim|x|^{-1-\alpha}$ 
$(0<\alpha \leq 2)$ (Padash et al., 2019). Evidence accumulation in the LF model can therefore be written as,

$$
X(t+\Delta t)=X(t)+v \cdot \Delta t+\operatorname{Stable}\left(\alpha, \beta=0, \gamma=\frac{1}{\sqrt{2}}, \delta=0\right) \cdot \Delta t^{\frac{1}{\alpha}}
$$

In Equation 2, $\alpha, \beta, \gamma$, and $\delta$ are the parameters of an $\alpha$-stable distribution, where $\alpha \in(0,2]$ is the stability index (Lévy index), $\beta \in[-1,1]$ is the skewness parameter, $\gamma>0$ is the scale parameter, and $\delta$ is the shift parameter that can be any real number. In the LF model, the distribution of accumulation noise is $\alpha$-stable with fixed parameters $\beta=0, \gamma=\frac{1}{\sqrt{2}}$, and $\delta=0$ (Gikhman and Skorokhod, 1975; Samorodnitsky and Taqqu, 1994) and $\alpha$ is the free parameter which is constrained to $1 \leq \alpha \leq 2$ (i.e. $p^{\alpha}(x)=\operatorname{Stable}\left(\alpha, \beta=0, \gamma=\frac{1}{\sqrt{2}}, \delta=0\right)$, and $\left.1 \leq \alpha \leq 2\right)$. When $\alpha=2$, the accumulation noise is Gaussian, and the model is equivalent to the DDM (Equation 1). When $\alpha=1$, the accumulation noise is Cauchy distributed. Accumulation dynamics for the standard DDM and the LF model with different $\alpha$ values are shown in Figure 2. 


\section{Lèvy Flights Model}

$$
\alpha=1.1
$$

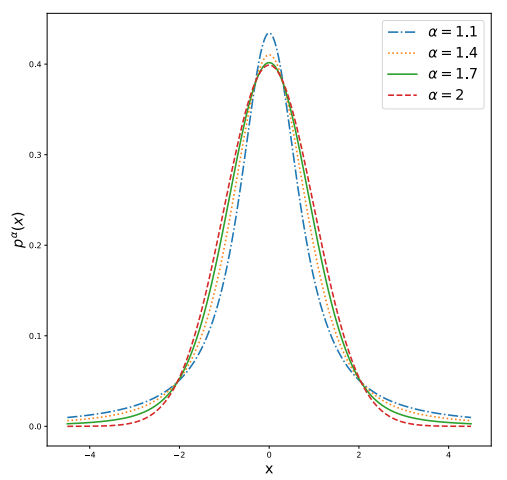

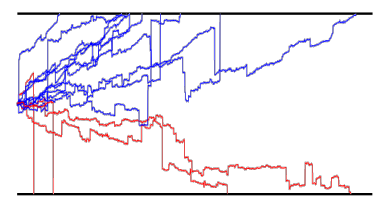

$\alpha=1.7$

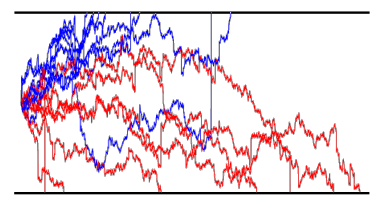

$\alpha=1.4$

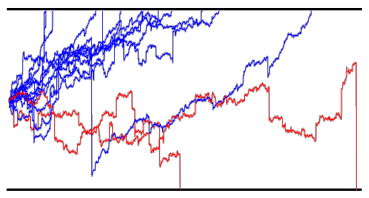

$\alpha=2.0$

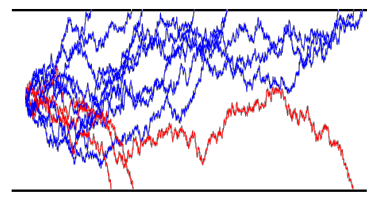

Figure 2. Plot of sample paths for the Lévy Flights model for different values of $\alpha$. In the left panel, the noise distribution of the accumulation process is presented for different values of $\alpha$. The right panels show sample accumulation paths under different values of $\alpha$. When $\alpha=2$, accumulation noise is Gaussian, and the model is equivalent to the standard diffusion decision model. As $\alpha$ takes on values lower than 2, accumulation noise becomes more variable, and large jumps in the accumulation process, depicted as sudden spikes in the accumulation paths, become more commonplace. The reader is referred to the online version of this article for a colored version of the figure.

To date, the LF model has only been applied sparingly in psychological research. The domains in which the model has proved useful, however, is quite broad, having been applied to memory search and retrieval processes (Patten et al., 2020), semantic memory search processes (Montez et al., 2015; Rhodes and Turvey, 2007), spatial memory (Kerster et al., 2016), perception in typically developing children and children with autism spectrum disorder (Liberati et al., 2017), and animal foraging (Reynolds, 2012). As mentioned above, Voss and colleagues have recently used the LF model as a model of decision-making (Voss et al., 2019; Wieschen et al., 2020), arguing for several potential benefits over the DDM, which we now summarize.

First, Voss et al. (2019) showed that LF models can provide highly accurate accounts of fast error patterns in data. In the DDM, fast errors have been previously explained in terms of start-point variability 
(Ratcliff and Rouder, 1998b; Smith et al., 2014). While start-point variability has good psychological plausibility-it can be related theoretically to sequential effects in stimulus presentation and/or responding (e.g., Rabbitt, 1969), an account that has received support from cognitive modeling (e.g., (Bode et al., 2012))-reliance on between-trial variability parameters has been criticized due to relatively poor parameter recovery properties (Lerche and Voss, 2016; Voss et al., 2019). The LF model can account for errors that are, on average, faster than correct responses without between-trial variability in start-point, attributing them to large jumps that occur early in evidence accumulation that quickly move the process toward the incorrect absorbing boundary. This account can be investigated in more detail by examining correlations between the $\alpha$ parameter and overall error rate (Wieschen et al., 2020).

Second, extending the applications to data exhibiting fast errors, the LF model may be useful for examining time-pressured decisions, where fast decisions are more important than accurate ones. In these paradigms, a decision-maker can choose among three types of strategies: (1) maintaining a low fixed evidence threshold, (2) implementing a collapsing evidence threshold (Cisek et al., 2009; Drugowitsch et al., 2012; Ditterich, 2006; Hawkins et al., 2015; Thura et al., 2012; Zhang et al., 2014), or (3) capitalizing on sudden jumps during evidence accumulation. A low fixed evidence threshold leads to many incorrect decisions in difficult conditions and is therefore not a good option (Evans et al., 2020). A collapsing threshold overcomes the problem of an unacceptably high error rate in difficult conditions, but does not produce fast errors (Evans et al., 2020). Taking advantage of sudden jumps in evidence allows the participant to reach an acceptable level of overall accuracy in this type of time-pressured environment while also accounting for fast errors. Wieschen et al. (2020) found high positive correlations between response time (RT) and the $\alpha$ parameter, as well as between overall accuracy (ACC) and the $\alpha$ parameter: the shorter the response time (or the faster the response), the smaller the $\alpha$ parameter, which implies that sudden jumps in evidence accumulation contributed to these faster decisions.

Third, jumps in evidence accumulation can be related to the "jumping to a conclusion" phenomenon (McKay et al., 2006), which is not readily achieved within a DDM framework. The heavy tails in the accumulation process outlined by the LF model are controlled by the value of the $\alpha$ parameter. Psychologically, lower values of $\alpha$ could correspond to a higher probability of suddenly accumulating an extreme amount of evidence (Wieschen et al., 2020). This phenomenon may occur in groups with high impulsivity, whose decision strategies may be more naturally modeled as a LF process due to lack of stability. Sudden accumulation of evidence might also be interpretable as a consequence of changes in the allocation of attentional resources that arise through the use of a hypothesis testing strategy (e.g., toggling between different decision strategies when judging a multi-attribute stimulus; cf. Lamberts, 1995).

Notwithstanding the potential advantages of using the LF model as a generalized form of the DDM, it is unclear whether situations where the LF outperforms the DDM are best attributed to the LF model providing a more accurate characterization of within-trial noise, or simply to increased model flexibility. Although Voss and colleagues have argued for the former (Voss et al., 2019; Wieschen et al., 2020), support for the LF model as a decision-making model has come primarily from assessing goodness of fit against other competing models (e.g., the standard DDM and various collapsing threshold models), and not from a 
more detailed theoretical analysis of the critical $\alpha$ parameter.

We address this issue by exploring Voss et al.'s (2019) conjecture that jumps in evidence accumulation may be due to sudden on-the-fly shifts of attention that may reflect instability in the decision strategy (or the capacity to execute the strategy) adopted by the observer. We argue that if the jumps in evidence accumulation assumed by the LF model can be psychologically interpreted in this way, we should be able to observe systematic changes in $\alpha$, as people become more experienced performing a task or become more familiar with the stimuli they are presented with. That is, the prevalence of sudden jumps in evidence accumulation should progressively reduce as people settle on a consistent decision strategy for performing a task and/or grow more adept at parsing and encoding stimuli in a way that supports effective task performance. This means that people's early decision performance might best be modeled using an $\alpha$ value that is less than 2, but with increasing experience over the course of an experiment, approaches a value of 2, approximating the behavior of the standard DDM. If, on the other hand, $\alpha$ cannot be psychologically interpreted in this way-if it enables better fits to data by simply increasing flexibility-we should not observe any systematic changes in the parameter with experience. To further explore the issue of model flexibility, we also consider whether the $\alpha$ parameter trades off with other between-trial variability parameters in the standard DDM (e.g., variability in start-point).

We structure the rest of the article as follows. We first provide a more detailed mathematical overview of the LF model. We then report simulation results exploring the behavior of the $\alpha$ parameter as it relates to summary measures of performance (viz. accuracy and RT), and how these relationships depend on specific combinations of stimulus and decision factors (i.e., different combinations of drift rate and threshold). We then report a re-analysis of data from Evans and Brown (2017) that enables tracking of the $\alpha$ parameter (and other LF model parameters) as a function of task experience. In their study, Evans and Brown (2017) analyzed people's performance in a motion discrimination task using the DDM, showing that people set overly cautious decision thresholds at the beginning of the task, but relax their thresholds with experience in a way that approaches an optimal setting (i.e., one that maximizes the rate of reward on the task). Reductions in decision threshold coincided with systematic increases in drift rates - the latter reflecting better encoding and extraction of motion information from the stimulus - and so one might predict that if the $\alpha$ parameter reflects shifting attention (or instability in executing a decision strategy), its value

should systematically change in a way that reflects the gradual refinement of (or convergence toward) a stable encoding and decision strategy. Comparing changes in $\alpha$ over time with corresponding changes in other between-trial variability parameters (i.e., $s v, s z r$, and $s t$ ) allows us to judge whether $\alpha$ is simply acting as a surrogate for one of these parameters, which would suggest that improvements in fit afforded by the LF model might reflect greater model flexibility rather than a more accurate description of psychological processing.

\section{Lévy Flights Model}

Mathematically, the Lévy Flights model is a generalized continuous-time random walk process, a sequential sampling model (Voss et al., 2019) that uses an $\alpha$-stable jump length distribution (or Lévy distribution) 
(Gnedenko and Kolmogorov, 1954) with a long-tailed, power-law asymptote $\lambda(x) \sim|x|^{-1-\alpha}(0<\alpha \leq 2)$ to describe noise in the accumulation process (Padash et al., 2019, 2020). Therefore, information accumulation in LF model can be formulated as follows:

$$
\left\{\begin{array}{l}
X(0)=z \\
X(t+\Delta t)=X(t)+v \cdot \Delta t+e \Delta t^{\frac{1}{\alpha}}, \quad e \sim p^{\alpha}(x), \quad 1<\alpha<2
\end{array}\right.
$$

where $p^{\alpha}(x)=\operatorname{Stable}\left(\alpha, \beta=0, \gamma=\frac{1}{\sqrt{2}}, \delta=0\right), v$ is the drift rate, and the process terminates whenever $X(t) \geq a$ or $X(t) \leq 0$. By considering Equation 3 the process can be considered as a continuous time process $v t+Z_{t}$, in which:

$$
Z_{t}=\frac{e_{1}+\cdots+e_{c t}}{c t^{\frac{1}{\alpha}}}
$$

Thus, the Fourier Transform of the probability density function of the location of the accumulated evidence total is obtained as follows:

$$
\hat{p}(k, t)=E\left[e^{-i k\left(v t+Z_{t}\right)}\right]=e^{-i k v t+r(i k)^{\alpha}+q(-i k)^{\alpha}},
$$

where $r$ represents the probability of jumping upward, and $q$ is the probability of jumping downward. Moreover, it can be concluded that the $\hat{p}(k, t)$ satisfies the following differential equation (Meerschaert and Sikorskii, 2011):

$$
\frac{d \hat{p}(x, t)}{d t}=-i k v+r\left(i k^{\alpha}\right)+q\left(-i k^{\alpha}\right)
$$

By applying inverse Fourier Transform on both side of Eq. 6 the following space fractional partial differential equation is obtained (Meerschaert and Sikorskii, 2011; Padash et al., 2019):

$$
\frac{\partial}{\partial t} p(x, t)+v \frac{\partial}{\partial x} p(x, t)=D_{x}^{\alpha} p(x, t)
$$

and by regarding the starting and termination conditions of the process, the following initial and boundary conditions are obtained:

$$
p(x, 0)=\delta(x-z), \quad p(a, t)=p(0, t)=0 .
$$

The fraction derivative in Eq. 7 (i.e. $\left.D_{x}^{\alpha}\right)$ is defined as follows:

$$
D_{x}^{\alpha} p(x, t)=\frac{-1}{2 \cos \frac{\alpha \pi}{2}}\left({ }_{-\infty}^{R} D_{x}^{\alpha} p(x, t)+{ }_{x}^{R} D_{\infty}^{\alpha} p(x, t)\right)
$$

where ${ }_{-\infty}^{R} D_{x}^{\alpha}$ and ${ }_{x}^{R} D_{\infty}^{\alpha}$ are the left and right Riemann-Liouville fractional derivatives and have the following definitions (Ding and Li, 2017; Hadian Rasanan et al., 2020):

$$
\begin{aligned}
{ }_{-\infty}^{R} D_{x}^{\alpha} p(x, t) & =\frac{1}{\Gamma(2-\alpha)} \frac{d^{2}}{d x^{2}} \int_{-\infty}^{x} \frac{p(\xi, t)}{(x-\xi)^{\alpha-1}} d \xi \\
{ }_{x}^{R} D_{\infty}^{\alpha} p(x, t) & =\frac{1}{\Gamma(2-\alpha)} \frac{d^{2}}{d x^{2}} \int_{x}^{\infty} \frac{p(\xi, t)}{(\xi-x)^{\alpha-1}} d \xi
\end{aligned}
$$


By considering Equation 7, the probability of the location of the accumulated evidence total at time $t$ can be obtained by solving the space fractional partial differential equation. Thus, by approximating the solution of this equation the survival probability of the decision process can be obtained as:

$$
S(t)=\int_{0}^{a} p(x, t) d x
$$

which determines whether the location of the accumulated evidence total is still somewhere between the absorbing boundaries at time $t$, or if the process has already terminated at one of them.

\section{Simulation Study}

In order to investigate a potential psychological interpretation of the $\alpha$ parameter in the LF model, we first examine the behavior of $\alpha$ in different parts of the parameter space of the LF model through simulation. Here, we examine relationships between $\alpha$ and summary measures of simulated data (i.e., accuracy and RT) as a function of different combinations of stimulus and decision factors (i.e., combinations of drift rate and threshold). For these simulations, parameter values were sampled from the following priors:

$$
\begin{aligned}
v & \sim \mathcal{N}(2.5,1), \\
z r & \sim \mathcal{U}(0.3,0.7), \\
a & \sim \mathcal{U}(0.5,3),
\end{aligned}
$$

where $\sim$ means "is distributed as". The non-decision time was fixed for all simulations at $t_{0}=0.3$. We varied $\alpha$ from $\{1.05,1.1,1.15, \cdots, 1.95,2\}$ in steps of 0.05 . In total, we simulated 400 experiments with different parameter values in which each experiment contained 700 trials. The data simulation procedure is summarized in Algorithm 1. For each set of fixed parameters (i.e. $\{v, a, z r\})$ an experiment with 700 trials is simulated with all $\alpha$ values. Thus for each parameter set we have $\left\{v, a, z r, \alpha_{1}, A C C_{1}, R T_{1}\right\} \cdots\left\{v, a, z r, \alpha_{20}, A C C_{20}, R T_{20}\right\}$. Then the each parameter set is mapped to the correlation values of the $\alpha=\{1.05,1.1,1.15, \cdots, 1.95,2\}$ with Accuracy $=\left\{A C C_{1}, \cdots, A C C_{20}\right\}$ and $M R T=\left\{\operatorname{mean}\left(R T_{1}\right), \cdots, \operatorname{mean}\left(R T_{20}\right)\right\}$.

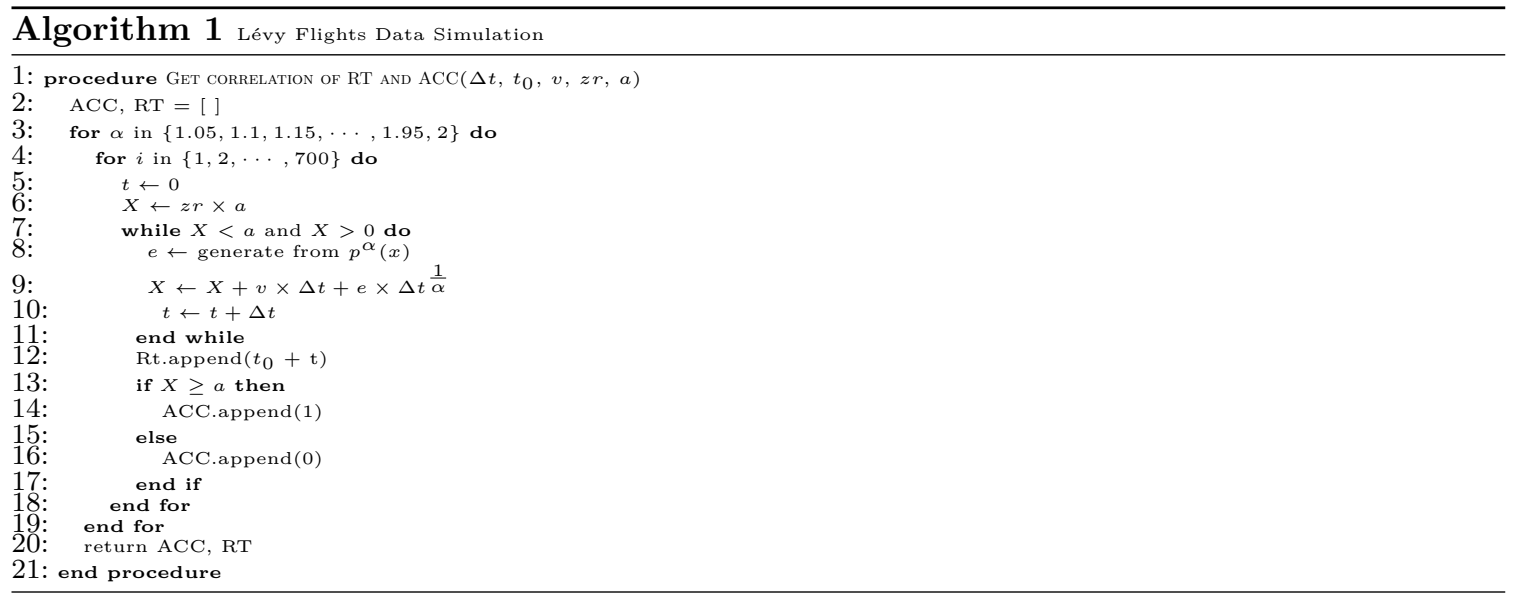




\section{Simulation results}

To get a clearer sense of how the $\alpha$ parameter relates to summary measures of performance (i.e., accuracy and RT) in different regions of the parameter space of the LF model, we plot the correlation between $\alpha$ and either accuracy or RT as a function of the best-fitting estimate of other core model parameters (e.g., mean drift rate, boundary separation, and start-point). Figure 3 shows the pattern of correlations with RT and Figure 4 shows correlations with accuracy in different regions of the parameter space. The effect of drift rate and threshold on the correlation between $\alpha$ and RT is demonstrated in Figure 3a. It is clear that both the drift rate and threshold affect this correlation. When both the drift rate and the threshold have high values, the correlation tends to 1 and when both of them have low values the correlation tends to -1 . This means when the threshold and the drift rate are high, response times increase as the prevalence of jumps in the accumulation process is reduced (i.e., $\alpha \longrightarrow 2$ ). In other words, when a cautious decision threshold is adopted, then a more stable accumulation process has a longer survival time. On the other hand, when the drift rate and threshold are low, a lower prevalence of jumps in evidence accumulation is also associated with faster RTs. This means when the threshold and the drift rate have low values, a more stable accumulation process finishes sooner.

Similarly, Figure 3b presents the pattern of bias and threshold effects on the $\alpha$-RT correlation. In this figure, the influence of threshold is clear - correlations between $\alpha$ and RT change from negative to positive as threshold increases - but at high values of the threshold, the effect is moderated by bias. In addition, Figure 3c shows that there is no obvious pattern for the $\alpha$-RT correlation in the bias and drift rate

space. Finally, Figure 3d represents that the $\alpha$-RT correlation has symmetric behavior in the parameter space, producing a bimodal distribution of strong positive and negative correlations. 

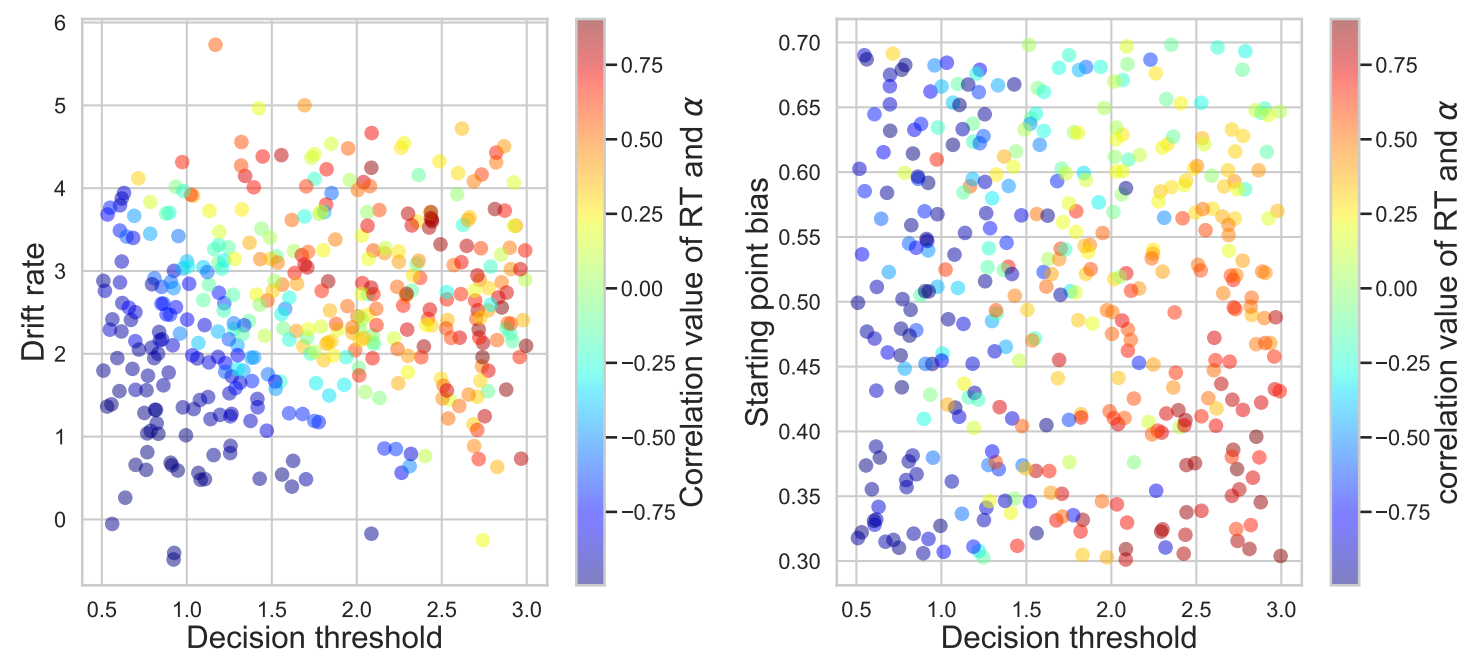

(a)

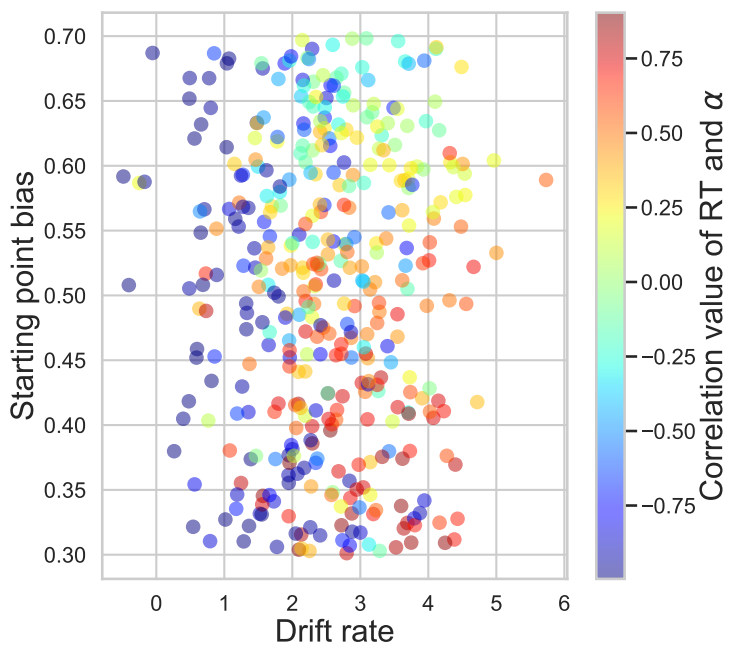

(c) (b)

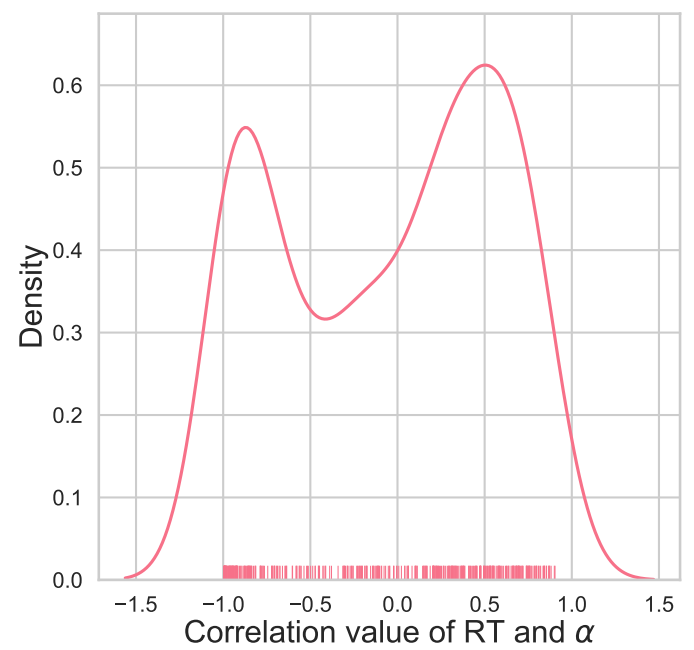

(d)

Figure 3. Correlations between $\alpha$ and RT in different regions of the parameter space. (a) The pattern of $\alpha$-RT correlation with respect to threshold and drift rate. (b) The pattern of $\alpha$-RT correlation with respect to threshold and bias. (c) The pattern of $\alpha$-RT correlation with respect to bias and drift rate. (d) Distribution of $\alpha$-RT correlation in the parameter space. We refer the reader onto the online version of this article for the color version of this figure. 
Figure 4 illustrates $\alpha$-ACC correlations. Unlike correlations with RT, $\alpha$ correlations with accuracy only appear to be affected by decision threshold, with no obvious systematic relationships with bias or drift rates. When thresholds are high, accuracy improves and is accompanied by reductions in jumps in the accumulation process (i.e., with a high decision threshold, a more stable accumulation process gives better accuracy). On the other hand, when the threshold is low, increases in accuracy are accompanied by increases in the prevalence of jumps in the accumulation process (i.e., with a low decision threshold, a less stable accumulation process gives better accuracy). Finally, Figure 4d presents that in almost all parts of the parameter space, accuracy improves as jumps in evidence accumulation become less prevalent. 

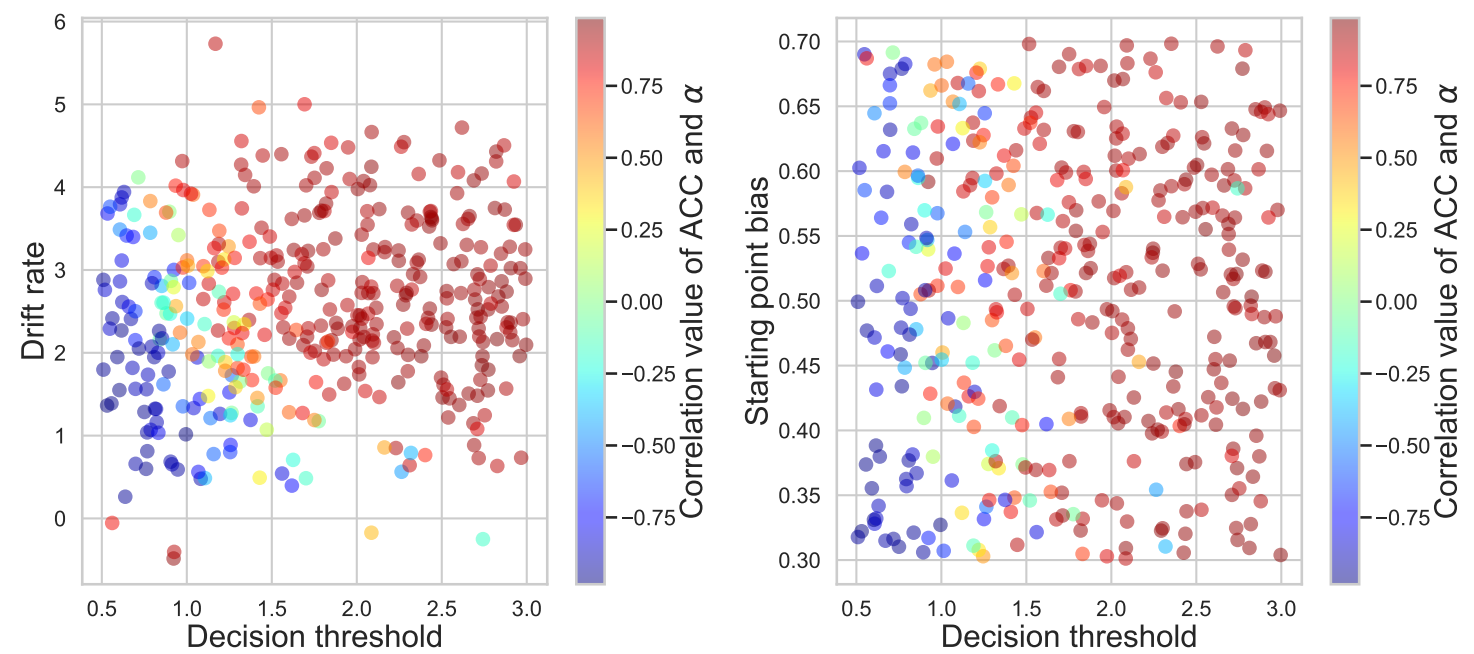

(a)

(b)
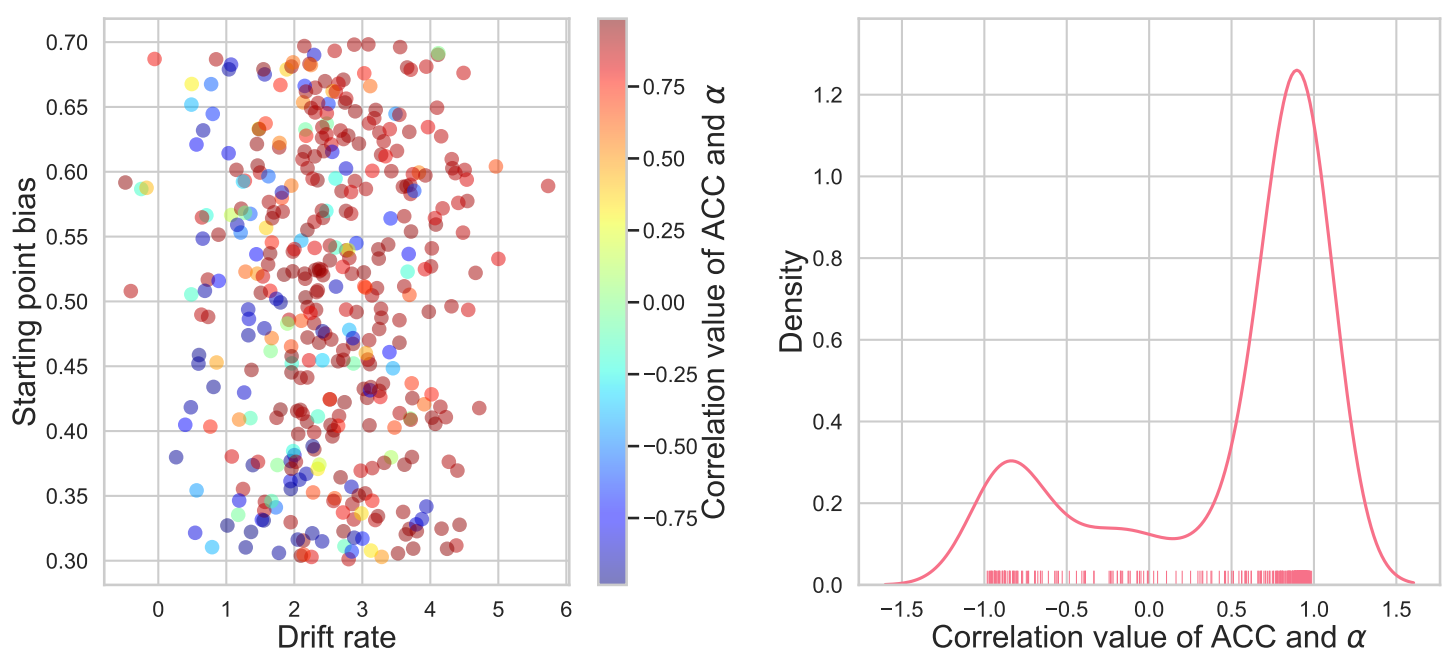

(c)

(d)

Figure 4. Correlations between $\alpha$ and accuracy (ACC) in different regions of the parameter space. (a) The pattern of $\alpha$-ACC correlation with respect to threshold and drift rate. (b) The pattern of $\alpha$-ACC correlation with respect to threshold and bias. (c) The pattern of $\alpha$-ACC correlation with respect to bias and drift rate. (d) Distribution of $\alpha$-ACC correlation in the parameter space. We refer the reader onto the online version of this article for the color version of this figure. 
Figure 5 illustrates the pattern of correlation between $\alpha$ and the reward rate, defined as the proportion of correct responses divided by the mean correct response time. When the decision threshold is low (i.e., approximately less than 1) $\alpha$ correlates negatively with the reward rate (i.e., higher reward rates are achieved when jumps in evidence accumulation are more prevalent). On the other hand, when the decision threshold is high (i.e., approximately greater than 1) $\alpha$ correlates positively with reward rate (i.e., higher reward rates are achieved when jumps in evidence accumulation are less prevalent).

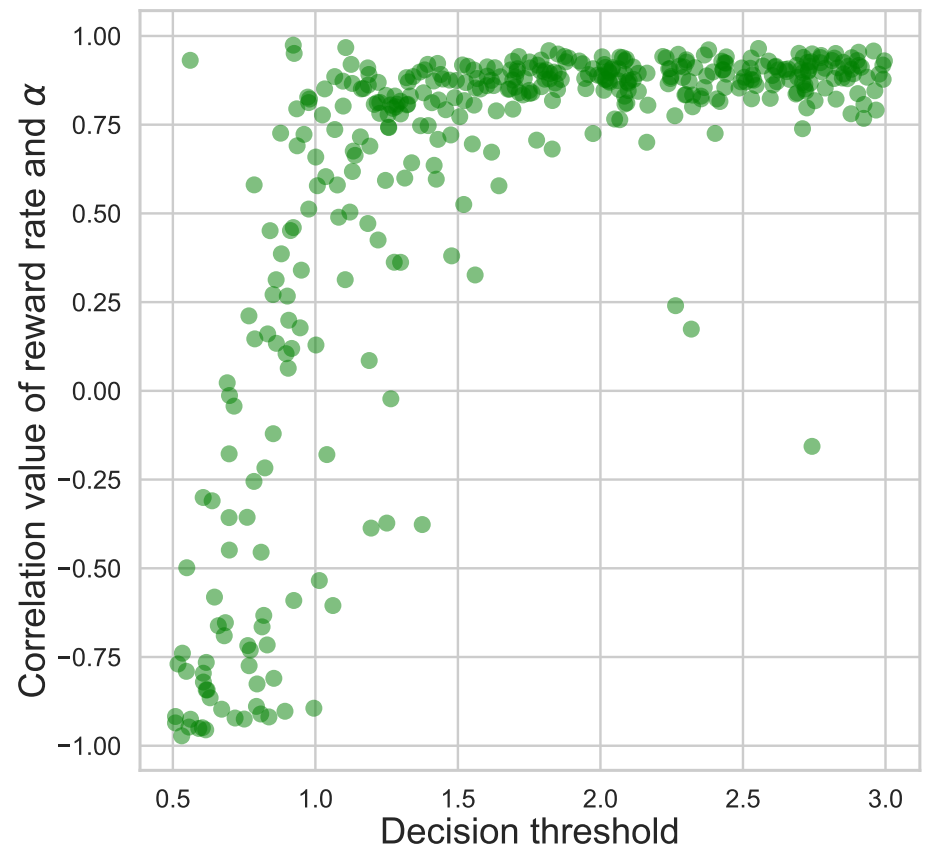

Figure 5. Correlations between $\alpha$ and reward rate as a function of threshold.

\section{Evans \& Brown (2017) Data Reanalysis}

We now present a reanalysis of data from a study by Evans and Brown (2017), using the LF model. Their study examined reward rate optimality in perceptual decision-making when people had either a fixed amount of time to complete the task, or a fixed number of trials to complete. For simplicity, our analysis focuses on the fixed-trial conditions from their study, in which participants completed a fixed number of trials in a motion discrimination task using random dot kinematogram stimuli (Roitman and Shadlen, 2002). On each trial, participants viewed an array of moving dots, a proportion of which moved coherently in one direction. Participants were randomly assigned to one of three conditions that differed in terms of the level of feedback provided at the end of each trial block. In the 'low information' condition, participants were only informed 
that the block had been completed. In the 'medium information' condition, participants were informed about their performance in that block (i.e., how many points they accrued in the block, the time taken to complete the block, and the rate at which they accrued points). In the 'high information' condition, some hints on how participants could improve their performance was presented in addition to the summary provided in the medium information condition (i.e., participants were advised that responding faster/slower could adjust their performance in a way that increased the rate at which they accrued points). The key finding from the study was that people tend to perform non-optimally by being overly cautious. However, with highly informative feedback and practice on the task, people's performance rapidly approaches optimality.

\section{Fits of the Lévy Flights Model to Human Data}

Following Evans and Brown (2017), we removed participants with accuracy less than 70\%, yielding 30 participants with data that we modeled (i.e., 10 participants in the 'low information' condition, nine in the 'medium information' condition, and 11 in the 'high information' condition). Evans and Brown analyzed the data from each of the 24 blocks of trials using the DDM, yielding parameter estimates for each individual block. For our analysis, the relatively low number of trials per block $(\mathrm{N}=40)$ presented difficulties in parameter recovery for the LF model, and so we elected to collapse the data into four larger trial epochs. Each epoch consisted of six blocks of the original design (e.g., Epoch 1 consisted of data from trial blocks 1-6 of the Evans and Brown study). Each epoch in our analysis comprised $\mathrm{N}=240$ trials.

Our main interest was to examine changes in the LF jump parameter, $\alpha$, over the course of the task. If this parameter reflects instability in selecting or executing a decision strategy, as conjectured by Voss et al. (2019), we would expect to see systematic changes in $\alpha$ in the practice data of Evans and Brown (2017). To this end, we compared four versions of the LF model, each instantiating different assumptions about the latent processes involved in decision making.

The models were fitted to the data from the high, medium, and low information conditions such that all model parameters were allowed to vary across conditions (i.e., different parameter estimates across different conditions). For each model, we estimated a decision threshold for each trial epoch $\left(a_{1}, a_{2}, a_{3}, a_{4}\right)$. Following (Evans and Brown, 2017), we also estimated a single drift rate $(v)$ for the entire experiment. We also estimated a single relative starting point bias $\left(z r=\frac{z}{a}\right)$, and a single non-decision time $\left(t_{0}\right)$ for the entire experiment. In the model of primary interest, the $\alpha$ varying model, we estimated a separate $\alpha$ parameter for each trial epoch. We did not estimate any sources of between-trial parameter variability for this model (i.e., $s v, s z r$, and $s t$ were all fixed to 0 ). We also considered several control models that all assumed a fixed level of $\alpha$ across the experiment, but allowed a different between-trial variability parameter to differ across trial epochs. The rationale for the control models was to determine whether the jump parameter in the LF model is simply mimicking the effects of one of the between-trial variability parameters, which confer some additional flexibility to the standard DDM. Specifically, we fitted three control models, the drift rate variability model, the start-point variability model, and non-decision time variability model, which each allowed their namesake parameter, $s v, s z r$, or $s t$, respectively to vary across trial epochs. To the extent that any systematic changes in $\alpha$ are mirrored in the other between-trial variability parameters, we can conclude 
that $\alpha$ primarily contributes flexibility to the LF model without having a clear psychological interpretation that is distinct from existing model parameters. Table 1 summarizes the free parameters for the four models fitted to the data.

\section{Table 1}

Summary of free parameters for the four models fitted to preregistered data by Evans and Brown (2017).

\begin{tabular}{ccc}
\hline Model & Free parameters & \# Free parameters \\
\hline$\alpha$ Varying Model & $\left\{v, t_{0}, z, a_{1}, a_{2}, a_{3}, a_{4}, \alpha_{1}, \alpha_{2}, \alpha_{3}, \alpha_{4}\right\}$ & 11 \\
Drift Variability Model & $\left\{v, z, t_{0}, \alpha, a_{1}, a_{2}, a_{3}, a_{4}, s v_{1}, s v_{2}, s v_{3}, s v_{4}\right\}$ & 12 \\
Start-Point Variability Model & $\left\{v, t_{0}, z, \alpha, a_{1}, a_{2}, a_{3}, a_{4}, s z r_{1}, s z r_{2}, s z r_{3}, s z r_{4}\right\}$ & 12 \\
Non-Decision Time Variability Model & $\left\{v, t_{0}, z, \alpha, a_{1}, a_{2}, a_{3}, a_{4}, s t_{1}, s t_{2}, s t_{3}, s t_{4}\right\}$ & 12 \\
\hline
\end{tabular}

For LF models, the lack of a closed-form likelihood function typically impedes Bayesian inference methods (Fengler et al., 2020; Voss et al., 2019). A notable paradigm of approximating the likelihood function is approximate Bayesian computing (ABC; Turner and Van Zandt, 2012). There are various ABC algorithms for likelihood approximation, but recently, some researchers have combined neural network approaches with traditional ABC algorithms and have improved them (Fengler et al., 2020; Radev et al., 2020). Previously, a convolutional neural network (CNN) was developed by Radev et al. (2020) to approximate the likelihood function. This network can approximate the parameters of a stochastic process by learning a large number of simulations of that process. We fit the four variants of the LF models described above using a deep inference algorithm based on the CNN described by Radev et al. (2020). This neural network approach learns approximate likelihoods for the LF models, allowing fast posterior sampling with only a one-off cost for model simulations that are amortized for future inference. Parameter estimation for each of the four LF models was achieved by training its own dedicated CNN. For example, for the non-decision time variability model, we generated 28000 data-sets with 240 trials of the LF model, and the network is trained using these data-sets. Each network had 5 layers and the size of filters in each layer were 64, 64, 128,128 , and 128, respectively. Additionally, each network consists of one channel for each trial epoch (i.e., four channels in total). The architecture of the utilized network is presented in Figure 6. 
Deep Inference Network Architecture

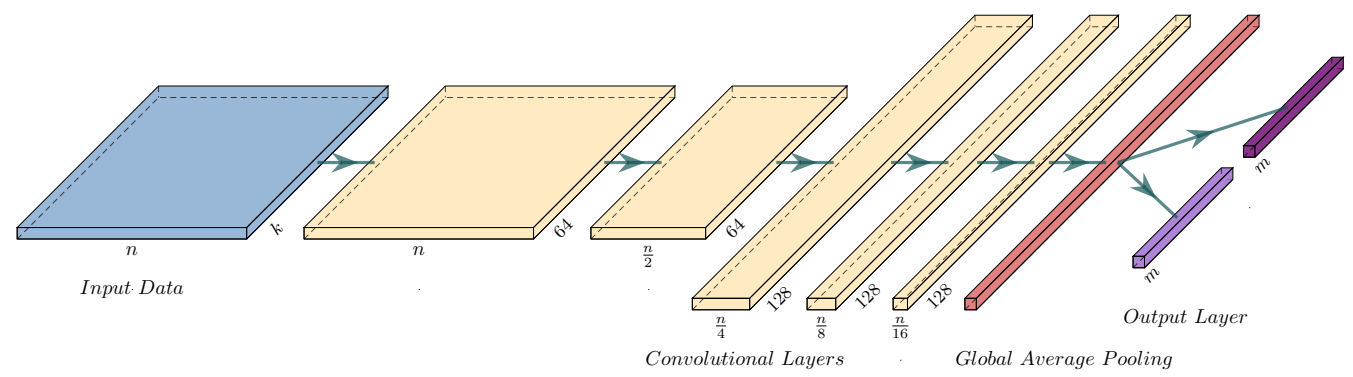

Figure 6. The architecture of the Deep Inference neural network which we used for parameter estimation in which $m$ denotes the number of parameters that the network is estimating, $k$ denotes the number of epochs, and $n$ denotes the size of input data.

The model estimates a drift rate, a non-decision time, a bias for the experiment, and four $\alpha$ and four decision threshold parameters (one for each trial epoch).

\section{Modeling results}

We now report the results of fitting the LF models to the individual data from each condition of the Evans and Brown (2017) study. We report summaries of the parameter estimates in the main text, and more detailed information about parameter estimates at the individual level in the supplementary materials.

We first consider the quality of fit for the $\alpha$ varying model to the data from the three information conditions. Figure 7 plots model predictions against observed data for the RT distribution data for correct responses (top panels), error responses (middle panels), and accuracy (bottom panel). The model provides a reasonable account of the data, though there is a general tendency for the model to predict slower correct RTs than are observed empirically, and to overestimate accuracy. 

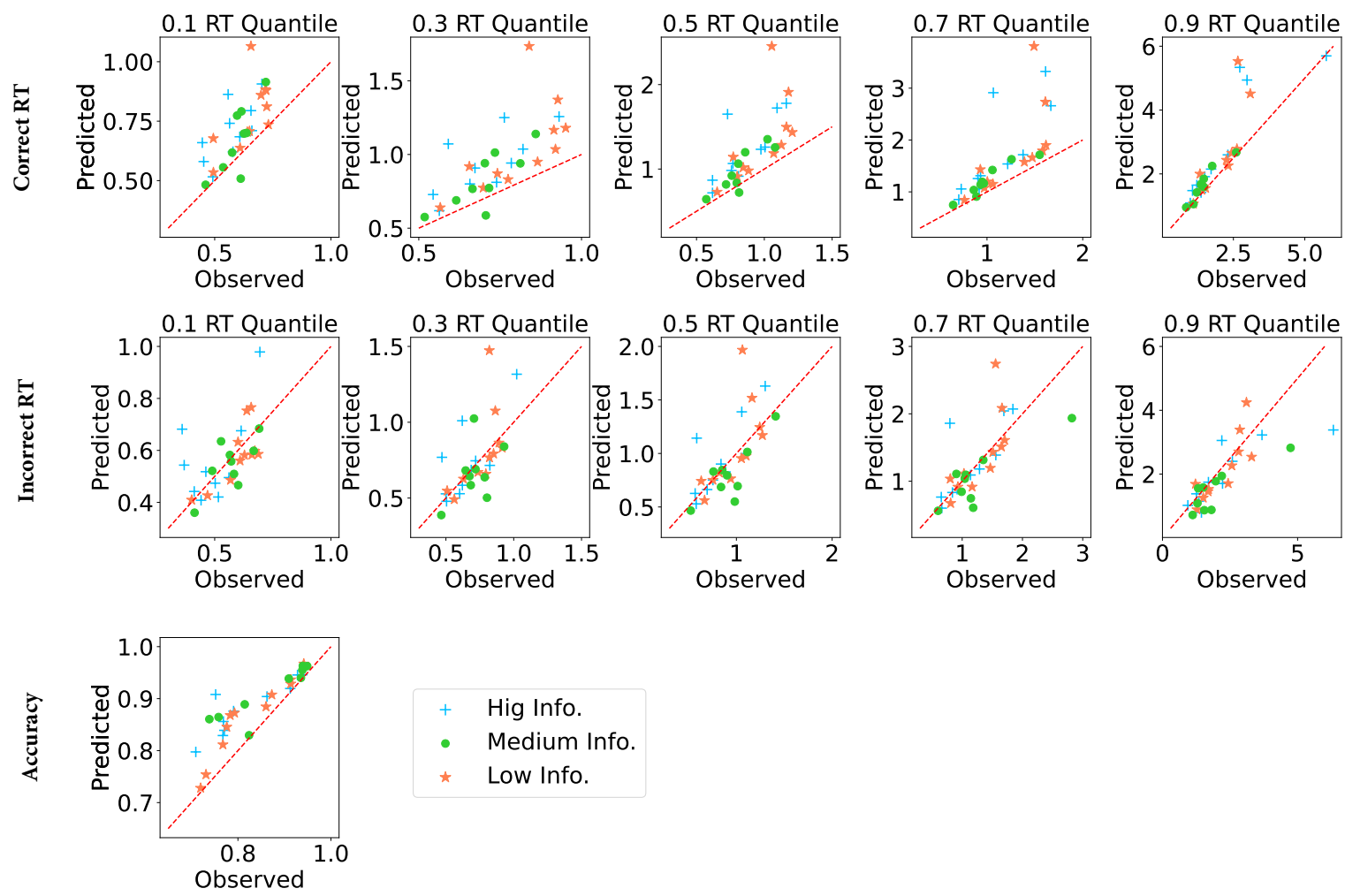

Hig Info.

- Medium Info.

* Low Info.

Figure 7. Predictions of the $\alpha$ varying model against observed data in different conditions for correct RTs (top panels), error RT (middle panels), and accuracy (bottom panel). Across columns, panels in the top two rows show response times for the $0.1,0.3,0.5,0.7$, and 0.9 distribution quantiles. Each point shows one participant.

Figure 8 shows changes in the estimates of $\alpha$ across trial epochs for each condition. A regression analysis reveals a significant pattern of increase only for the high information condition (Table 2 also shows the results of similar regression analyses for the control models and how their namesake parameters vary across trial epochs).

Figure 8 shows how the jump parameter, $\alpha$, varies across trial epochs in each condition. In the 'low' and 'medium' information conditions, changes in $\alpha$ are not clear-cut across trial epochs. In the 'high' information condition, however, there are clear and systematic increases in $\alpha$ across trial epochs, suggesting 
greater stability in strategy selection and/or execution with increasing practice. Indeed, the pattern of increasing $\alpha$ values in this condition means that the behavior of the model more closely approximates the standard DDM with increasing practice. If $\alpha$ tends to increase with practice, it is perhaps unsurprising why the DDM has proved so successful: in highly practiced participants, whose data are often used to test the standard model, within-trial accumulation noise will be well characterized by a Gaussian process (or equivalently, an $\alpha$-stable distribution, where $\alpha=2$ ).
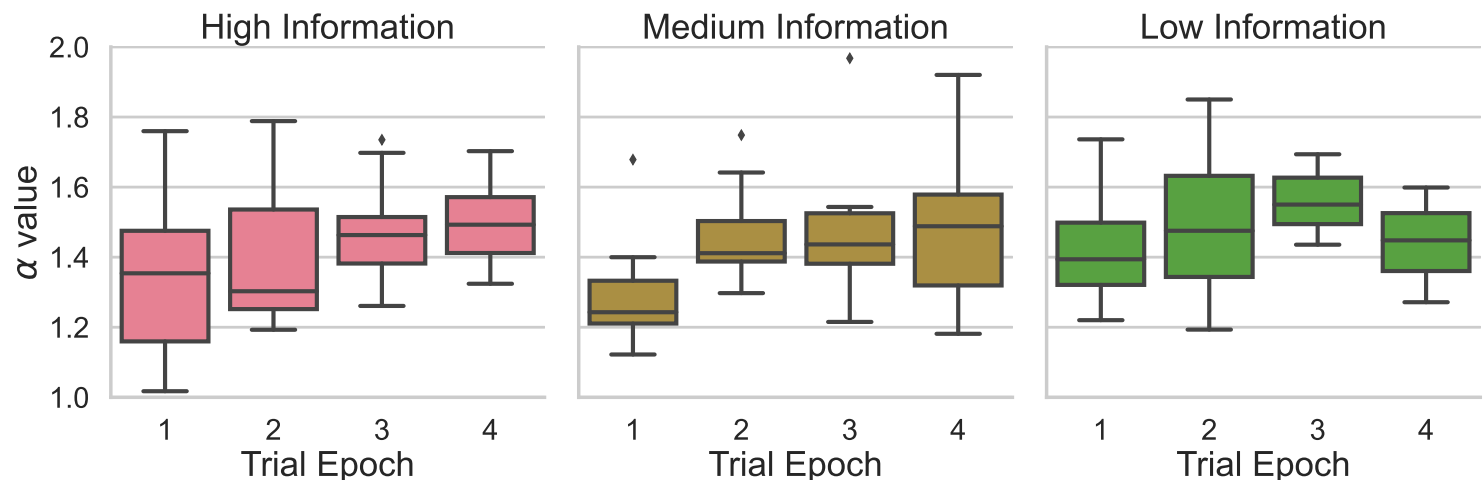

Figure 8. Boxplots depicting changes in the $\alpha$ parameter across trial epochs for the $\alpha$ varying model in the High (left panel), Medium (middle panel), and Low Information (right panel) conditions.

We note that the changes in $\alpha$ did not come at the expense of the changes in decision threshold reported by Evans and Brown (2017). Figure 9 shows systematic reductions in decision threshold across trial epochs, replicating their core result.
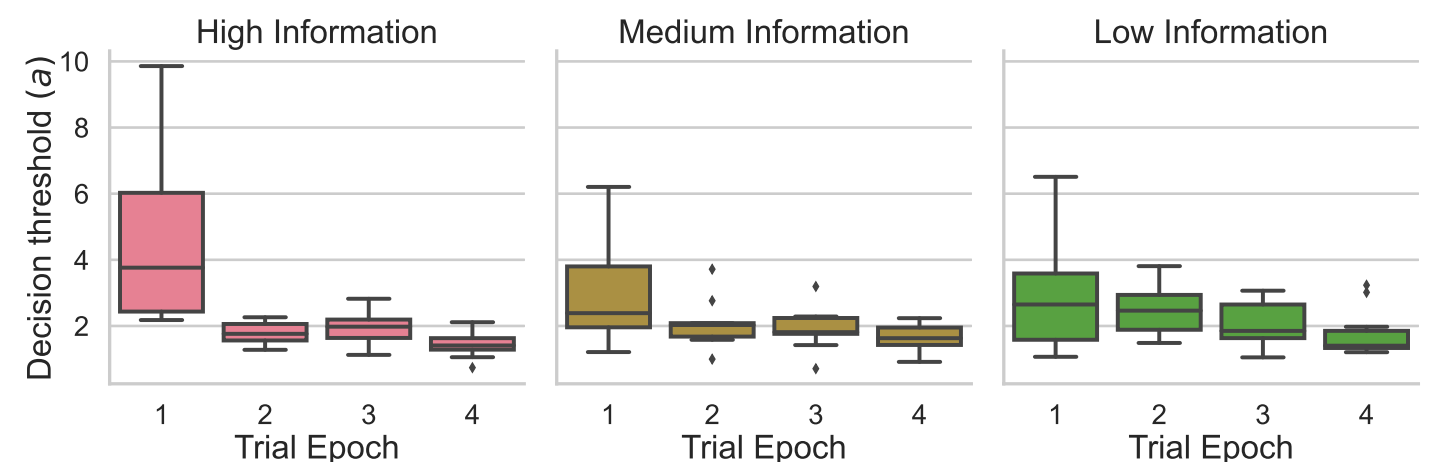

Figure 9. Boxplots depicting changes in the decision threshold (a) parameter across trial epochs for the $\alpha$ varying model in the High (left panel), Medium (middle panel), and Low Information (right panel) conditions. 
Figure 10, shows correlations between the $\alpha$ parameter and decision threshold parameter $(a)$. There are significant negative correlations between decision threshold $(a)$ and $\alpha$ in the 'medium' and 'high' information conditions, and a marginally significant negative correlation in the 'low' information condition.
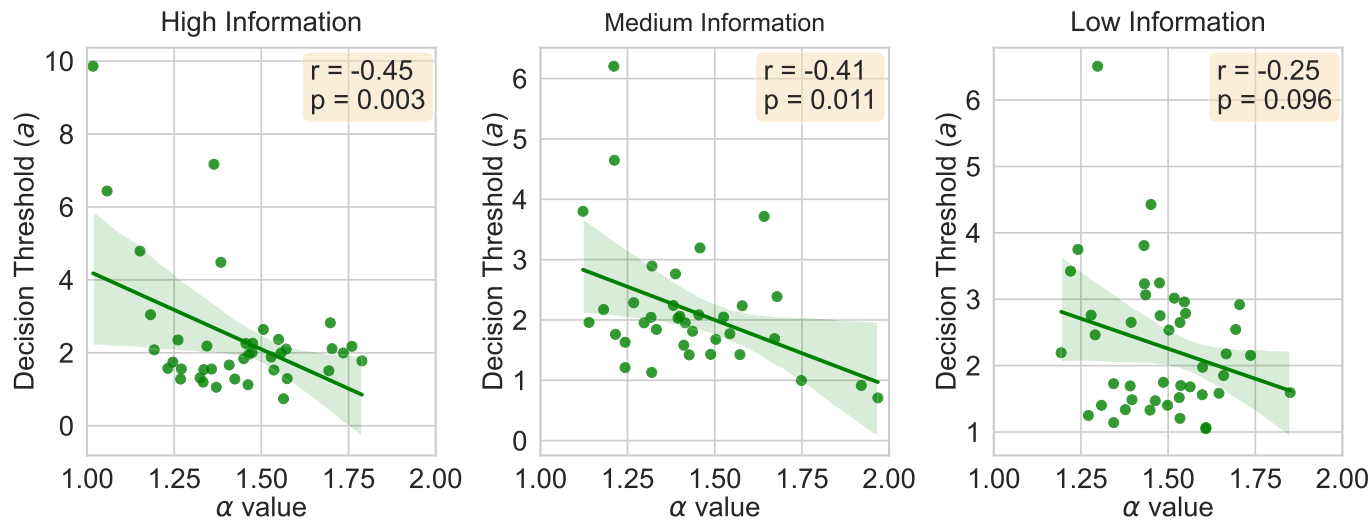

Figure 10. Correlations between $\alpha$ and decision threshold (a) for the High (left), Medium (middle), and Low Information (right) conditions. The shaded regions show 95\% confidence interval.

We next considered the performance of the three control models that allowed either drift rate variability $(s v)$, start-point variability $(s z r)$, or non-decision time variability $(s t)$ to differ across epochs. Changes in each model's namesake parameter across trial epochs are shown in Figure 11. It is clear from the bottom panels of the figure that there are no systematic changes in non-decision time variability across trial epochs. This suggests that there is no tradeoff between $\alpha$ and non-decision time variability. However, there appear to be systematic changes in drift rate variability (top panels) as well as start-point variability (middle panels) as a function of practice, which raises concerns about whether the changes in $\alpha$ reported for the $\alpha$ varying model are simply mimicking changes in these between-trial variability parameters that are available to the standard DDM.

Turning first to the drift variability model, there is a decreasing trend in drift rate variability, $s v$, across trial epochs in both the 'medium' and 'high' information conditions which are also reported in Table 2. This decreasing pattern is only significant in the 'medium' condition. One interpretation of this trend is that it reflects increased consistency in how the stimulus is encoded as people become more practiced with the task. This interpretation aligns well with the interpretation of $\alpha$ proposed by Voss et al. (2019) (i.e., that it reflects consistent strategy use and/or the capacity to reliably execute a given decision strategy). It is possible, then, that the changes in $\alpha$ seen with the $\alpha$ varying model are actually mimicking reductions in drift rate variability. Based on the model selection results reported in Table 3, and the relatively poor performance of the drift variability model, it appears that the degree of mimicry between $\alpha$ and $s v$ is only partial. Given that changes in $s v$ are insufficient to explain the effects of gradual increases in $\alpha$ with practice, 
we tentatively conclude that changes in $\alpha$ may reflect more stable reliance on a well-learned decision strategy, consistent with the claims of Voss et al. (2019), and that these changes are not restricted to consistency in strategy selection or application across trials.

Turning to the start-point variability model, there is a clear and significant increasing trend in start-point variability, szr across trial epochs in the all 'low', 'medium', and 'high' information conditions (i.e. see Table 2). The overall pattern of change, especially in the high information condition, is similar to the changes observed in $\alpha$ for the $\alpha$ varying model. The effect of increasing start-point variability, however, runs counter to the effect of increasing $\alpha$ in the LF model, and so we think it is unlikely that the changes in these model parameters observed here reflect any meaningful tradeoff. Specifically, start-point variability is important for allowing the standard DDM to fit patterns of data where error responses are, on average, faster than correct responses (Ratcliff and Rouder, 1998b). While fits to this fast-error pattern have been used to support the LF model (Voss et al., 2019), these fits require lower values of the $\alpha$ parameter. The increases in $\alpha$ shown by the $\alpha$ varying model imply a progressively lower rate of fast errors with practice, whereas the increasing pattern of $s z r$ shown by the start-point variability model implies an increasing rate of fast errors with practice. On balance, we conclude that changes in start-point variability do not provide a viable explanation of changes in the $\alpha$ parameter.

Overall, we conclude that the $\alpha$ varying model was the best performing model we considered. Model selection based on both AIC and BIC further support this (Table 3). Parameter estimates for each model are shown in Table 4. 

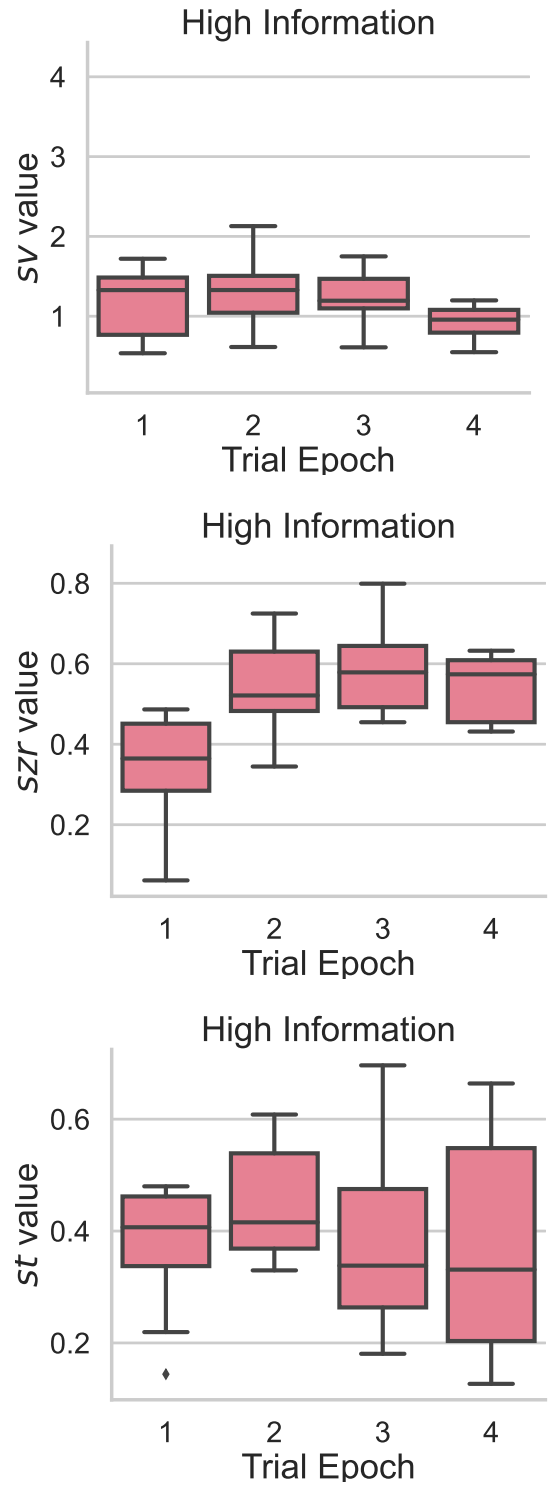

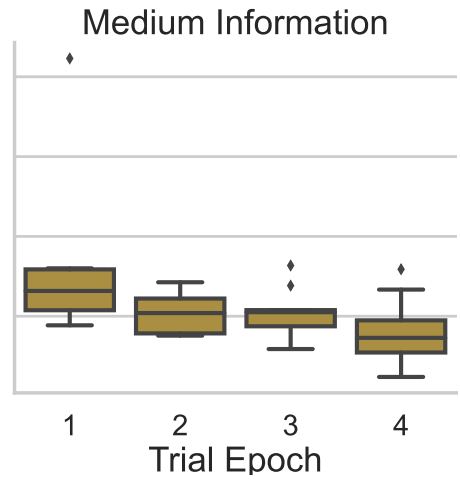

Medium Information

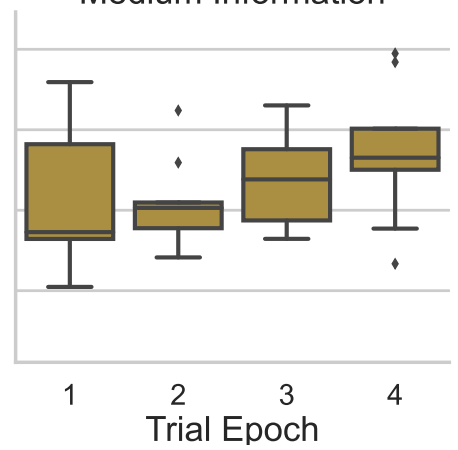

Medium Information

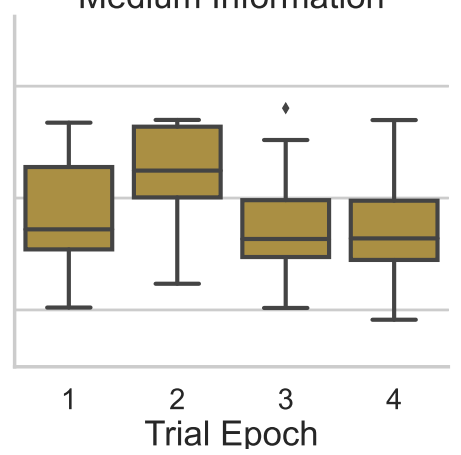

Low Information

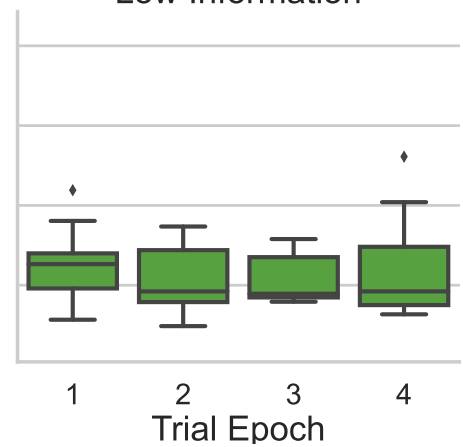

Low Information

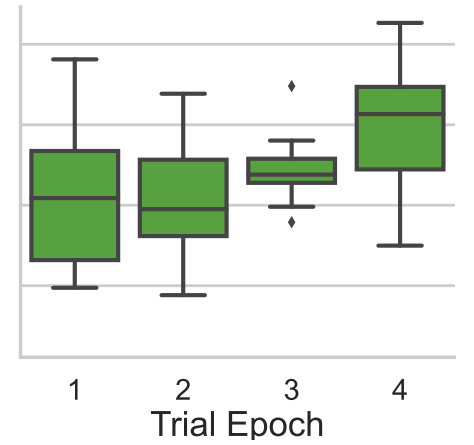

Low Information

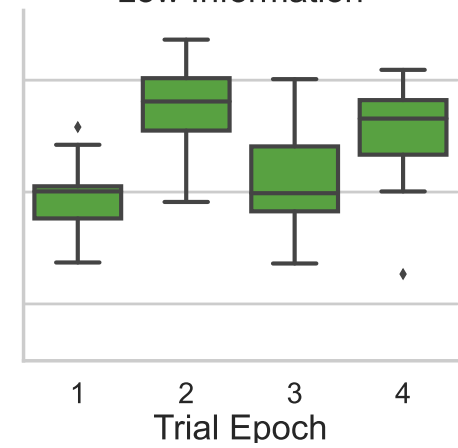

Figure 11. Boxplots depicting changes in between trial variability parameters of the drift variability model (top panel), start-point variability model (middle panel), and non-decision time variability model (bottom panel) for the High (left panel), Medium (middle panel), and Low Information (right panel) conditions across trial epochs. 
Table 2

Regression coefficient for model's namesake parameters.

\begin{tabular}{cccc}
\hline Model & High Info. & Medium Info. & Low Info. \\
\hline$\alpha$ Varying Model & $0.36^{*}$ & 0.32 & 0.08 \\
Drift Variability Model & -0.22 & $-0.40^{*}$ & -0.01 \\
Start-Point Variability Model & $0.49^{* *}$ & $0.33^{*}$ & $0.39^{* *}$ \\
Non-Decision Time Variability Model & -0.05 & -0.15 & 0.20 \\
\hline
\end{tabular}

Note. ${ }^{*}$ p-value $<0.05,{ }^{* *}$ p-value $<0.01$.

\section{Table 3}

Fitting performance of the all models. The best performing model for each fit index (log likelihood, $A I C$, and BIC) is shown in bold.

\begin{tabular}{clllc}
\hline & Model 1 & Model 2 & Model 3 & Model 4 \\
\hline $\log$ likelihood & $\mathbf{- 6 1 6 2 4}$ & -70157 & -90672 & -164413 \\
AIC & $\mathbf{1 2 3 5 7 8}$ & 140674 & 181704 & 329187 \\
BIC & $\mathbf{1 2 5 5 1 4}$ & 142786 & 183817 & 331299 \\
\hline
\end{tabular}

Note. Model $1=\alpha$ Varying Model; Model $2=$ Drift Variability Model; Model $3=$ Start-Point Variability Model; Model 4 = Non-Decision Time Variability Model. 
Table 4

Estimated parameters of the models for each condition (i.e. mean (variance)).

\begin{tabular}{|c|c|c|c|c|c|c|c|c|c|c|c|c|c|}
\hline Model & Condition & $a_{1}$ & $a_{2}$ & $a_{3}$ & $a_{4}$ & $v$ & $z r$ & $t_{0}$ & $\alpha_{1}$ & $\alpha_{2}$ & $\alpha_{3}$ & $\alpha_{4}$ & \\
\hline \multirow{4}{*}{ Model 1} & High Info. & $\begin{array}{l}4.51 \\
(6.72)\end{array}$ & $\begin{array}{l}1.79 \\
(0.09)\end{array}$ & $\begin{array}{l}1.91 \\
(0.26)\end{array}$ & $\begin{array}{l}1.43 \\
(0.15)\end{array}$ & $\begin{array}{l}1.30 \\
(0.37)\end{array}$ & $\begin{array}{l}0.50 \\
(0.01)\end{array}$ & $\begin{array}{l}0.46 \\
(0.01)\end{array}$ & $\begin{array}{l}1.33 \\
(0.05)\end{array}$ & $\begin{array}{l}1.39 \\
(0.03)\end{array}$ & $\begin{array}{l}1.47 \\
(0.02)\end{array}$ & $\begin{array}{l}1.50 \\
(0.01)\end{array}$ & - \\
\hline & Medium Info. & $\begin{array}{l}3.00 \\
(2.56)\end{array}$ & $\begin{array}{l}2.09 \\
(0.59)\end{array}$ & $\begin{array}{l}1.91 \\
(0.45)\end{array}$ & $\begin{array}{l}1.62 \\
(0.20)\end{array}$ & $\begin{array}{l}1.71 \\
(0.35)\end{array}$ & $\begin{array}{l}0.52 \\
(0.02)\end{array}$ & $\begin{array}{l}0.47 \\
(0.006)\end{array}$ & $\begin{array}{l}1.29 \\
(0.02)\end{array}$ & $\begin{array}{l}1.46 \\
(0.02)\end{array}$ & $\begin{array}{l}1.46 \\
(0.04)\end{array}$ & $\begin{array}{l}1.48 \\
(0.05)\end{array}$ & - \\
\hline & Low Info. & $\begin{array}{l}2.81 \\
(2.69)\end{array}$ & $\begin{array}{l}2.46 \\
(0.56)\end{array}$ & $\begin{array}{l}2.10 \\
(0.42)\end{array}$ & $\begin{array}{l}1.78 \\
(0.49)\end{array}$ & $\begin{array}{l}1.12 \\
(0.18)\end{array}$ & $\begin{array}{l}0.51 \\
(0.01)\end{array}$ & $\begin{array}{l}0.49 \\
(0.004)\end{array}$ & $\begin{array}{l}1.42 \\
(0.02)\end{array}$ & $\begin{array}{l}1.49 \\
(0.04)\end{array}$ & $\begin{array}{l}1.55 \\
(0.006)\end{array}$ & $\begin{array}{l}1.44 \\
(0.01)\end{array}$ & - \\
\hline & & $a_{1}$ & $a_{2}$ & $a_{3}$ & $a_{4}$ & $v$ & $z r$ & $t_{0}$ & $\alpha$ & $s v_{1}$ & $s v_{2}$ & $s v_{3}$ & $s v_{4}$ \\
\hline \multirow{4}{*}{ Model 2} & High Info. & $\begin{array}{l}5.81 \\
(16.36)\end{array}$ & $\begin{array}{l}1.76 \\
(0.16)\end{array}$ & $\begin{array}{l}1.88 \\
(0.50)\end{array}$ & $\begin{array}{l}1.68 \\
(0.35)\end{array}$ & $\begin{array}{l}2.31 \\
(1.11)\end{array}$ & $\begin{array}{l}0.37 \\
(0.01)\end{array}$ & $\begin{array}{l}0.41 \\
(0.01)\end{array}$ & $\begin{array}{l}1.29 \\
(0.01)\end{array}$ & $\begin{array}{l}1.16 \\
(0.20)\end{array}$ & $\begin{array}{l}1.34 \\
(0.21)\end{array}$ & $\begin{array}{l}1.25 \\
(0.11)\end{array}$ & $\begin{array}{l}0.92 \\
(0.04)\end{array}$ \\
\hline & Medium Info. & $\begin{array}{l}3.18 \\
(3.08)\end{array}$ & $\begin{array}{l}2.12 \\
(0.42)\end{array}$ & $\begin{array}{l}2.06 \\
(0.46)\end{array}$ & $\begin{array}{l}2.01 \\
(0.21)\end{array}$ & $\begin{array}{l}2.61 \\
(0.63)\end{array}$ & $\begin{array}{l}0.40 \\
(0.009)\end{array}$ & $\begin{array}{l}0.44 \\
(0.008)\end{array}$ & $\begin{array}{l}1.28 \\
(0.01)\end{array}$ & $\begin{array}{l}1.57 \\
(1.04)\end{array}$ & $\begin{array}{l}1.03 \\
(0.05)\end{array}$ & $\begin{array}{l}1.04 \\
(0.09)\end{array}$ & $\begin{array}{l}0.82 \\
(0.18)\end{array}$ \\
\hline & Low Info. & $\begin{array}{l}3.29 \\
(4.74) \\
\end{array}$ & $\begin{array}{l}2.46 \\
(0.54)\end{array}$ & $\begin{array}{l}2.34 \\
(0.60) \\
\end{array}$ & $\begin{array}{l}2.06 \\
(0.54) \\
\end{array}$ & $\begin{array}{l}1.89 \\
(0.39) \\
\end{array}$ & $\begin{array}{l}0.39 \\
(0.004)\end{array}$ & $\begin{array}{l}0.46 \\
(0.003)\end{array}$ & $\begin{array}{l}1.32 \\
(0.02) \\
\end{array}$ & $\begin{array}{l}1.23 \\
(0.22)\end{array}$ & $\begin{array}{l}1.05 \\
(0.20) \\
\end{array}$ & $\begin{array}{l}1.06 \\
(0.09)\end{array}$ & $\begin{array}{l}1.21 \\
(0.41) \\
\end{array}$ \\
\hline & & $a_{1}$ & $a_{2}$ & $a_{3}$ & $a_{4}$ & $v$ & $z r$ & $t_{0}$ & $\alpha$ & $s z r 1$ & $s z r 2$ & $s z r 3$ & $s z r 4$ \\
\hline \multirow{4}{*}{ Model 3} & High Info. & $\begin{array}{l}4.96 \\
(6.06)\end{array}$ & $\begin{array}{l}1.80 \\
(0.17)\end{array}$ & $\begin{array}{l}1.75 \\
(0.43)\end{array}$ & $\begin{array}{l}1.69 \\
(0.21)\end{array}$ & $\begin{array}{l}1.47 \\
(0.78)\end{array}$ & $\begin{array}{l}0.59 \\
(0.01)\end{array}$ & $\begin{array}{l}0.53 \\
(0.01)\end{array}$ & $\begin{array}{l}1.17 \\
(0.01)\end{array}$ & $\begin{array}{l}0.33 \\
(0.02)\end{array}$ & $\begin{array}{l}0.54 \\
(0.01)\end{array}$ & $\begin{array}{l}0.58 \\
(0.01)\end{array}$ & $\begin{array}{l}0.54 \\
(0.006)\end{array}$ \\
\hline & Medium Info. & $\begin{array}{l}3.02 \\
(1.96)\end{array}$ & $\begin{array}{l}2.09 \\
(0.22)\end{array}$ & $\begin{array}{l}1.78 \\
(0.50)\end{array}$ & $\begin{array}{l}1.86 \\
(0.20)\end{array}$ & $\begin{array}{l}1.76 \\
(0.70)\end{array}$ & $\begin{array}{l}0.59 \\
(0.02)\end{array}$ & $\begin{array}{l}0.56 \\
(0.005)\end{array}$ & $\begin{array}{l}1.13 \\
(0.01)\end{array}$ & $\begin{array}{l}0.41 \\
(0.02)\end{array}$ & $\begin{array}{l}0.41 \\
(0.01)\end{array}$ & $\begin{array}{l}0.46 \\
(0.01)\end{array}$ & $\begin{array}{l}0.54 \\
(0.02)\end{array}$ \\
\hline & Low Info. & $\begin{array}{l}3.04 \\
(3.69) \\
\end{array}$ & $\begin{array}{l}2.43 \\
(0.23) \\
\end{array}$ & $\begin{array}{l}2.11 \\
(0.33) \\
\end{array}$ & $\begin{array}{l}2.05 \\
(0.56) \\
\end{array}$ & $\begin{array}{l}1.14 \\
(0.34) \\
\end{array}$ & $\begin{array}{l}0.58 \\
(0.01)\end{array}$ & $\begin{array}{l}0.60 \\
(0.01)\end{array}$ & $\begin{array}{l}1.19 \\
(0.01) \\
\end{array}$ & $\begin{array}{l}0.42 \\
(0.03)\end{array}$ & $\begin{array}{l}0.41 \\
(0.02)\end{array}$ & $\begin{array}{l}0.48 \\
(0.007)\end{array}$ & $\begin{array}{l}0.59 \\
(0.02)\end{array}$ \\
\hline & & $a_{1}$ & $a_{2}$ & $a_{3}$ & $a_{4}$ & $v$ & $z r$ & $t_{0}$ & $\alpha$ & st1 & st2 & st3 & st4 \\
\hline \multirow{3}{*}{ Model 4} & High Info. & $\begin{array}{l}4.90 \\
(50.26)\end{array}$ & $\begin{array}{l}1.33 \\
(0.25)\end{array}$ & $\begin{array}{l}1.46 \\
(0.27)\end{array}$ & $\begin{array}{l}1.28 \\
(0.34)\end{array}$ & $\begin{array}{l}1.31 \\
(0.62)\end{array}$ & $\begin{array}{l}0.64 \\
(0.02)\end{array}$ & $\begin{array}{l}0.62 \\
(0.01)\end{array}$ & $\begin{array}{l}1.13 \\
(0.01)\end{array}$ & $\begin{array}{l}0.37 \\
(0.01)\end{array}$ & $\begin{array}{l}0.44 \\
(0.01)\end{array}$ & $\begin{array}{l}0.37 \\
(0.02)\end{array}$ & $\begin{array}{l}0.37 \\
(0.04)\end{array}$ \\
\hline & Medium Info. & $\begin{array}{l}3.20 \\
(3.39)\end{array}$ & $\begin{array}{l}1.96 \\
(0.89)\end{array}$ & $\begin{array}{l}1.66 \\
(0.43)\end{array}$ & $\begin{array}{l}1.71 \\
(0.42)\end{array}$ & $\begin{array}{l}1.75 \\
(1.05)\end{array}$ & $\begin{array}{l}0.67 \\
(0.02)\end{array}$ & $\begin{array}{l}0.63 \\
(0.02)\end{array}$ & $\begin{array}{l}1.14 \\
(0.01)\end{array}$ & $\begin{array}{l}0.36 \\
(0.01)\end{array}$ & $\begin{array}{l}0.43 \\
(0.01)\end{array}$ & $\begin{array}{l}0.35 \\
(0.01)\end{array}$ & $\begin{array}{l}0.34 \\
(0.01)\end{array}$ \\
\hline & Low Info. & $\begin{array}{l}2.79 \\
(4.89)\end{array}$ & $\begin{array}{l}2.03 \\
(0.76)\end{array}$ & $\begin{array}{l}1.89 \\
(1.11)\end{array}$ & $\begin{array}{l}1.62 \\
(0.77)\end{array}$ & $\begin{array}{l}0.99 \\
(0.23)\end{array}$ & $\begin{array}{l}0.57 \\
(0.02)\end{array}$ & $\begin{array}{l}0.62 \\
(0.006)\end{array}$ & $\begin{array}{l}1.19 \\
(0.02)\end{array}$ & $\begin{array}{l}0.39 \\
(0.004)\end{array}$ & $\begin{array}{l}0.54 \\
(0.007)\end{array}$ & $\begin{array}{l}0.41 \\
(0.009)\end{array}$ & $\begin{array}{l}0.50 \\
(0.01)\end{array}$ \\
\hline
\end{tabular}

Note. Model $1=\alpha$ Varying Model; Model $2=$ Drift Variability Model; Model 3 = Start-Point Variability Model; Model 4 = Non-Decision Time Variability Model.

\section{Discussion}

Our primary aim with the current study was to investigate the assumptions made by the LF model regarding within-trial evidence accumulation dynamics. Specifically, we provided a critical investigation of the psychological plausibility of the sudden large jumps in evidence accumulation that the model allows via its $\alpha$ parameter. While previous work has shown that the LF model provides a good fit to data exhibiting patterns of fast errors (Voss et al., 2019), it is not clear whether this is simply due to the increased flexibility afforded by the $\alpha$, or if the LF model provides a more accurate characterization of the underlying psychological process. Voss et al. (2019) proposed that $\alpha$ might be interpreted as reflecting the stability or consistency with which an individual selects or executes a given decision strategy (e.g., jumps in evidence accumulation, due to $\alpha$, may reflect sudden shifts in the source of evidence when people are less adept at 
parsing the stimulus). This conjecture was the subject of our investigation.

We reasoned that if $\alpha$ could be interpreted psychologically along the lines proposed by Voss et al. (2019), we should be able to observe systematic changes in this parameter, as people refine a decision strategy and become more experienced in executing it. That is, we might expect systematic reductions in the $\alpha$ parameter as a function of practice. We reanalyzed practice data from a study by Evans and Brown (2017), who showed different patterns of improvement as a function of how informative the performance feedback they received was. Our analysis of these data with the LF model showed that when $\alpha$ was free to vary as a function of practice, as in the $\alpha$ varying model, we saw systematic increases in $\alpha$, as a function of practice. This increasing pattern was most evident in the high information condition, where participants received the most guidance on how to refine their decision strategy over time. Our result coheres well with the conjecture of (Voss et al., 2019), as the high information condition would be where one would expect participants to have refined their decision strategy to the greatest degree.

We also considered whether the changes in $\alpha$ we observed could simply reflect a tradeoff with some other model parameter that is available to the standard DDM (viz. between-trial variability in either drift rate, starting point, or non-decision time). Our analysis showed that while there is some overlap between changes in $\alpha$ and changes in trial-to-trial variability in drift rate, $s v$, the changes in the drift rate variability parameter alone are insufficient to explain the benefits of allowing $\alpha$ to vary with practice.

How then should we view the LF model, and the assumptions it makes regarding the nature of evidence accumulation? On the one hand, our application of the model to the practice data of Evans and Brown (2017) suggests that systematic changes in $\alpha$ unfold in a manner that is sensible and theoretically consistent with our understanding of how task performance improves as a function of practice. It is therefore tempting to conclude that the jumps in evidence accumulation implied by the LF model are indeed real, and that the $\alpha$ parameter indexes something akin to the "stability" of a decision strategy. We believe such a conclusion would be premature, as, on the other hand, there is a broader question of whether the stability in applying, selecting, or executing a decision strategy over the course of an experiment is best represented as a within-trial noise parameter controlling moment-to-moment perturbations in evidence accumulation, or if it could be better characterized in some other way. If refinements of decision strategy serve to limit the noise that enters the decision process - perhaps by allowing observers to focus attention on the most relevant or diagnostic parts of a stimulus, or by rendering the observer less vulnerable to momentary distraction-then it is perhaps appropriate to interpret $\alpha$ as an index of decision stability. If, however, the evolution of decision strategies is more consistent with a process of selecting and testing candidate decision hypotheses, it would be more sensible to consider decision stability as a phenomenon that is best understood as occurring across trials, rather than varying over the course of a single decision within a trial. If decision instability is more akin to discrete selection of candidate response strategies across trials, then the early stages of practicewhere estimates of $\alpha$ are low - may better be characterized by a probability mixture of Wiener diffusion processes, characterized by drift rate distributions with different means and standard deviations. These more detailed comparisons await future research. 


\section{Conclusion}

For now, we think it is prudent to conclude that the $\alpha$ parameter in LF models can be interpreted as reflecting a psychologically meaningful construct (viz. decision stability). However, future research will need to carefully investigate whether the construct is best represented in formal models of decision-making as a between- or within-trial phenomenon. Our analysis of practice data using the LF model suggests that more diagnostic tests of the model may require participants who are less practiced on tasks, as individuals in the early stages of practice will be most likely to be trying to discern the best way to perform and will be the most open to trying a number of different approaches.

\section{Declarations}

\section{Funding}

The authors have declared that they did not have any financial support for this research.

\section{Conflict of Interest}

The authors have declared that they do not have any conflict of interest.

\section{Code availability}

All data and codes are available on: https://osf.io/fgupj

\section{References}

Bode, S., Sewell, D. K., Lilburn, S., Forte, J. D., Smith, P. L., and Stahl, J. (2012). Predicting perceptual decision biases from early brain activity. Journal of Neuroscience, 32(36):12488-12498.

Bogacz, R., Brown, E., Moehlis, J., Holmes, P., and Cohen, J. D. (2006). The physics of optimal decisionmaking: a formal analysis of models of performance in two-alternative forced-choice tasks. Psychological Review, 113(4):700-765.

Brown, S. and Heathcote, A. (2005). A ballistic model of choice response time. Psychological review, 112(1):117.

Brown, S. D. and Heathcote, A. (2008). The simplest complete model of choice response time: Linear ballistic accumulation. Cognitive psychology, 57(3):153-178.

Cisek, P., Puskas, G. A., and El-Murr, S. (2009). Decisions in changing conditions: the urgency-gating model. The Journal of Neuroscience, 29(37):11560-11571.

Ding, H. and Li, C. (2017). High-order numerical algorithms for riesz derivatives via constructing new generating functions. Journal of Scientific Computing, 71(2):759-784. 
Ditterich, J. (2006). Evidence for time-variant decision making. European Journal of Neuroscience, 24(12):3628-3641.

Drugowitsch, J., Moreno-Bote, R., Churchland, A. K., Shadlen, M. N., and Pouget, A. (2012). The cost of accumulating evidence in perceptual decision making. The Journal of Neuroscience, 32(11):3612-3628.

Evans, N. J., Bennett, A. J., and Brown, S. D. (2018). Optimal or not; depends on the task. Psychonomic bulletin \& review, 26:1027-1034.

Evans, N. J. and Brown, S. D. (2017). People adopt optimal policies in simple decision-making, after practice and guidance. Psychonomic Bulletin \& Review, 24(2):597-606.

Evans, N. J., Hawkins, G. E., and Brown, S. D. (2020). The role of passing time in decision-making. Journal of experimental psychology: learning, memory, and cognition, 46(2):316.

Evans, N. J. and Wagenmakers, E.-J. (2020). Evidence accumulation models: Current limitations and future directions. The Quantitative Methods for Psychology.

Fengler, A., Frank, M., Govindarajan, L., and Chen, T. (2020). Likelihood approximation networks (lans) for fast inference of simulation models in cognitive neuroscience. bioRxiv.

Fontanesi, L., Gluth, S., Spektor, M. S., and Rieskamp, J. (2019). A reinforcement learning diffusion decision model for value-based decisions. Psychonomic bulletin \& review, 26:1099-1121.

Gikhman, I. I. and Skorokhod, A. V. (1975). Theory of Stochastic Processes II. Berlin: Springer.

Gluth, S., Kern, N., Kortmann, M., and Vitali, C. L. (2020). Value-based attention but not divisive normalization influences decisions with multiple alternatives. Nature human behaviour, 4(6):634-645.

Gnedenko, B. V. and Kolmogorov, A. N. (1954). Limit Distributions for Sums of Random Variables. Cambridge, MA: Addison-Wesley.

Gold, J. I. and Shadlen, M. N. (2007). The neural basis of decision-making. Annual Review of Neuroscience, $30: 535-574$

Gomez, P., Ratcliff, R., and Perea, M. (2007). A model of the go/no-go task. Journal of Experimental Psychology: General, 136(3):389.

Hadian Rasanan, A. H., Bajalan, N., Parand, K., and Rad, J. A. (2020). Simulation of nonlinear fractional dynamics arising in the modeling of cognitive decision making using a new fractional neural network. Mathematical Methods in the Applied Sciences, 43(3):1437-1466.

Hawkins, G. E., Forstmann, B. U., Wagenmakers, E.-J., Ratcliff, R., and Brown, S. D. (2015). Revisiting the evidence for collapsing boundaries and urgency signals in perceptual decision-making. Journal of Neuroscience, 35(6):2476-2484.

Hawkins, G. E. and Heathcote, A. (2021). Racing against the clock: Evidence-based versus time-based decisions. Psychological Review, 128(2):222. 
Kerster, B. E., Rhodes, T., and Kello, C. T. (2016). Spatial memory in foraging games. Cognition, 148:85-96.

Krajbich, I., Armel, C., and Rangel, A. (2010). Visual fixations and the computation and comparison of value in simple choice. Nature Neuroscience, 13(10):1292-1298.

Krajbich, I., Lu, D., Camerer, C., and Rangel, A. (2012). The attentional drift-diffusion model extends to simple purchasing decisions. Frontiers in Psychology, 3:193.

Kvam, P. D. (2019). A geometric framework for modeling dynamic decisions among arbitrarily many alternatives. Journal of Mathematical Psychology, 91:14-37.

Lamberts, K. (1995). Categorization under time pressure. Journal of Experimental Psychology: General, $124(2): 161$.

Lerche, V. and Voss, A. (2016). Model complexity in diffusion modeling: Benefits of making the model more parsimonious. Frontiers in psychology, 7:1324-1326.

Liberati, A., Fadda, R., Doneddu, G., Congiu, S., Javarone, M. A., Striano, T., and Chessa, A. (2017). A statistical physics perspective to understand social visual attention in autism spectrum disorder. Perception, 46(8):889-913.

Mallahi-Karai, K. and Diederich, A. (2019). Decision with multiple alternatives: Geometric models in higher dimensions - the cube model. Journal of Mathematical Psychology, 93:102294.

Mallahi-Karai, K. and Diederich, A. (2021). Decision with multiple alternatives: Geometric models in higher dimensions - the disk model. Journal of Mathematical Psychology, 100:102493.

Matzke, D., Dolan, C. V., Logan, G. D., Brown, S. D., and Wagenmakers, E.-J. (2013). Bayesian parametric estimation of stop-signal reaction time distributions. Journal of Experimental Psychology: General, $142(4): 1047$.

Matzke, D., Hughes, M., Badcock, J. C., Michie, P., and Heathcote, A. (2017a). Failures of cognitive control or attention? the case of stop-signal deficits in schizophrenia. Attention, Perception, ES Psychophysics, 79:1078-1086.

Matzke, D., Love, J., and Heathcote, A. (2017b). A bayesian approach for estimating the probability of trigger failures in the stop-signal paradigm. Behavior Research Methods, 49:267-281.

McKay, R., Langdon, R., and Coltheart, M. (2006). Need for closure, jumping to conclusions, and decisiveness in delusion-prone individuals. The Journal of Nervous and Mental Disease, 194(6):422-426.

Meerschaert, M. M. and Sikorskii, A. (2011). Stochastic models for fractional calculus, volume 43. Walter de Gruyter.

Miletić, S., Boag, R. J., Trutti, A. C., Stevenson, N., Forstmann, B. U., and Heathcote, A. (2021). A new model of decision processing in instrumental learning tasks. Elife, 10:e63055. 
Montez, P., Thompson, G., and Kello, C. T. (2015). The role of semantic clustering in optimal memory foraging. Cognitive science, 39(8):1925-1939.

Padash, A., Chechkin, A. V., Dybiec, B., Magdziarz, M., Shokri, B., and Metzler, R. (2020). First passage time moments of asymmetric lévy flights. Journal of Physics A: Mathematical and Theoretical.

Padash, A., Chechkin, A. V., Dybiec, B., Pavlyukevich, I., Shokri, B., and Metzler, R. (2019). First-passage properties of asymmetric lévy flights. Journal of Physics A: Mathematical and Theoretical, 52(45):454004.

Patten, K. J., Greer, K., Likens, A. D., Amazeen, E. L., and Amazeen, P. G. (2020). The trajectory of thought: Heavy-tailed distributions in memory foraging promote efficiency. Memory E Cognition, 48:772-787.

Pedersen, M. L., Frank, M. J., and Biele, G. (2017). The drift diffusion model as the choice rule in reinforcement learning. Psychonomic bulletin \& review, 24:1234-1251.

Rabbitt, P. (1969). Psychological refractory delay and response-stimulus interval duration in serial, choiceresponse tasks. Acta Psychologica, 30:195-219.

Radev, S. T., Mertens, U. K., Voss, A., Ardizzone, L., and Köthe, U. (2020). Bayesflow: Learning complex stochastic models with invertible neural networks. IEEE Transactions on Neural Networks and Learning Systems, pages 1-15.

Radev, S. T., Mertens, U. K., Voss, A., and Köthe, U. (2020). Towards end-to-end likelihood-free inference with convolutional neural networks. British Journal of Mathematical and Statistical Psychology, $73(1): 23-43$.

Ratcliff, R. (1978). A theory of memory retrieval. Psychological review, 85(2):59.

Ratcliff, R. (2018). Decision making on spatially continuous scales. Psychological Review, 125(6):888-935.

Ratcliff, R., Huang-Pollock, C., and McKoon, G. (2018). Modeling individual differences in the go/no-go task with a diffusion model. Decision, 5(1):42-62.

Ratcliff, R. and Mckoon, G. (2008). The diffusion decision model: Theory and data for two-choice decision tasks. Neural Computation, 20:873-922.

Ratcliff, R. and Rouder, J. N. (1998a). Modeling response times for two-choice decisions. Psychological Science, 9:347-356.

Ratcliff, R. and Rouder, J. N. (1998b). Modeling response times for two-choice decisions. Psychological science, $9(5): 347-356$.

Ratcliff, R. and Smith, P. L. (2004). A comparison of sequential-sampling models for two choice reaction time. Psychological Review, 111:333-367.

Ratcliff, R., Smith, P. L., Brown, S. D., and McKoon, G. (2016). Diffusion decision model: Current issues and history. Trends in Cognitive Sciences, 20:260-281. 
Reynolds, A. (2012). Olfactory search behaviour in the wandering albatross is predicted to give rise to Lévy flight movement patterns. Animal Behaviour, 83(5):1225-1229.

Rhodes, T. and Turvey, M. T. (2007). Human memory retrieval as lévy foraging. Physica A: Statistical Mechanics and its Applications, 385(1):255-260.

Roe, R. M., Busemeyer, J. R., and Townsend, J. T. (2001). Multi-alternative decision field theory: A dynamic artificial neural network model of decision-making. Psychological Review, 108:370-392.

Roitman, J. D. and Shadlen, M. N. (2002). Response of neurons in the lateral intraparietal area during a combined visual discrimination reaction time task. The Journal of Neuroscience, 22(21):9475-9489.

Samorodnitsky, G. and Taqqu, M. S. (1994). Stable Non-gaussian Random Processes: Stochastic Models with Infinite Variance. New York: Chapman and Hall.

Sewell, D. K., Jach, H. K., Boag, R. J., and Heer, C. A. V. (2019). Combining error-driven models of associative learning with evidence accumulation models of decision-making. Psychonomic bulletin $E^{6}$ review, 26:868-893.

Sewell, D. K. and Smith, P. L. (2016). The psychology and psychobiology of simple decisions: Speeded choice and its neural correlates. In Neuroeconomics, pages 253-292. Springer.

Sewell, D. K. and Stallman, A. (2020). Modeling the effect of speed emphasis in probabilistic category learning. Computational Brain \& Behavior, 3(2):129-152.

Smith, P. L. (2016). Diffusion theory of decision making in continuous report. Psychological Review, 123(4):425-451.

Smith, P. L., Ratcliff, R., and Sewell, D. K. (2014). Modeling perceptual discrimination in dynamic noise: Time-changed diffusion and release from inhibition. Journal of Mathematical Psychology, 59:95-113.

Smith, P. L. and Vickers, D. (1988). The accumulator model of two-choice discrimination. Journal of Mathematical Psychology, 32(2):135-168.

Starns, J. J. and Ratcliff, R. (2012). Age-related differences in diffusion model boundary optimality with both trial-limited and time-limited tasks. Psychonomic bulletin E review, 19(1):139-145.

Stone, M. (1960). Models for choice-reaction time. Psychometrika, 25(3):251-260.

Thura, D., Beauregard-Racine, J., Fradet, C. W., and Cisek, P. (2012). Decision making by urgency gating: theory and experimental support. Journal of Neurophysiology, 108(11):2912-2930.

Tillman, G., Van Zandtc, T., and Loganb, G. D. (2020). Sequential sampling models without random between-trial variability: the racing diffusion model of speeded decision making. Psychonomic bulletin $E_{3}$ review.

Trueblood, J. S., Brown, S. D., and Heathcote, A. (2014). The multi-attribute linear ballistic accumulator model of context effects in multi-alternative choice. Psychological Review, 121:179-205. 
Turner, B. M. and Van Zandt, T. (2012). A tutorial on approximate bayesian computation. Journal of Mathematical Psychology, 56(2):69-85.

Usher, M. and McClelland, J. L. (2001). The time course of perceptual choice: the leaky, competing accumulator model. Psychological review, 108(3):550.

Usher, M. and McClelland, J. L. (2004). Loss aversion and inhibition in dynamical models of multialternative choice. Psychological Review, 111:757-769.

Voss, A., Lerche, V., Mertens, U., and Voss, J. (2019). Sequential sampling models with variable boundaries and non-normal noise: A comparison of six models. Psychonomic bulletin $\&$ review, 26(3):813-832.

Wagenmakers, E.-J., Van Der Maas, H. L., and Grasman, R. P. (2007). An ez-diffusion model for response time and accuracy. Psychonomic bulletin \& review, 14(1):3-22.

Wieschen, E. M., Voss, A., and Radev, S. (2020). Jumping to conclusion? a lévy flight model of decision making. TQMP, 16(2):120-132.

Zhang, S., Lee, M. D., Vandekerckhove, J., Maris, G., and Wagenmakers, E.-J. (2014). Time-varying boundaries for diffusion models of decision making and response time. Frontiers in psychology, 5:1364. 


\section{Supplementary Materials}

In the supplementary material, model prediction of the drift variability, start-point variability, and nondecision time variability models are presented first. Then the estimated threshold parameters for all four models are presented and finally, individual estimated parameters are illustrated.

Model prediction of drift variability, start-point variability, and non-decision time variability models

Model prediction for the three control models described in the main text are shown below. Figures A.1, A.2, and A.3 show the model prediction for the drift variability, start-point variability, and non-decision time variability models, respectively. 

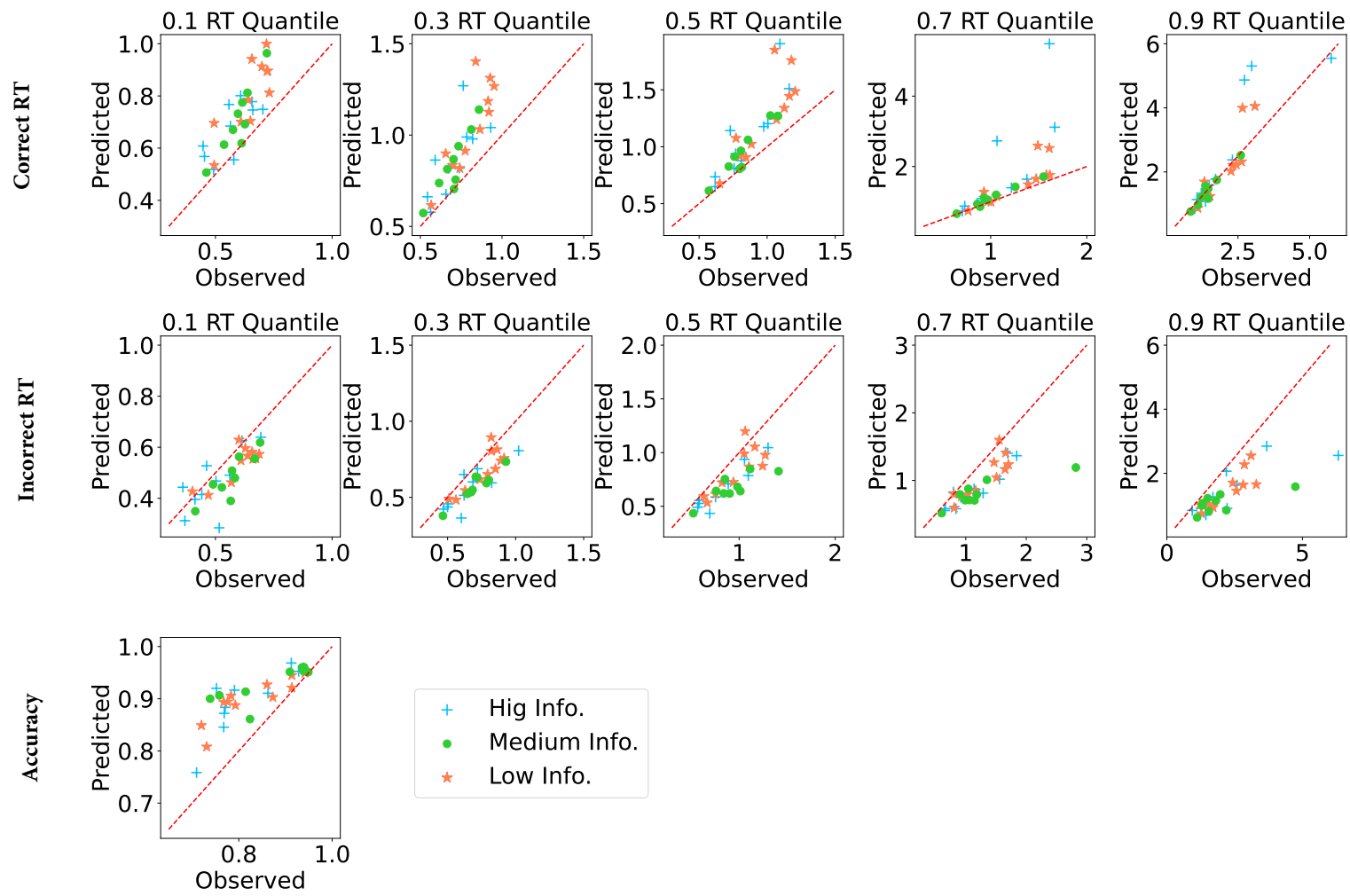

Hig Info.

- Medium Info.

* Low Info.

Figure A.1. Prediction of drift variability model for different conditions. Top panel shows correct reaction time for in 10-th, 30-th, 50-th, 70-th, and 90-th quantile. Middle panel shows same quatiles for incorrect reaction times. Button panel illustrate the accuracy prediction. Each point shows one participant. 

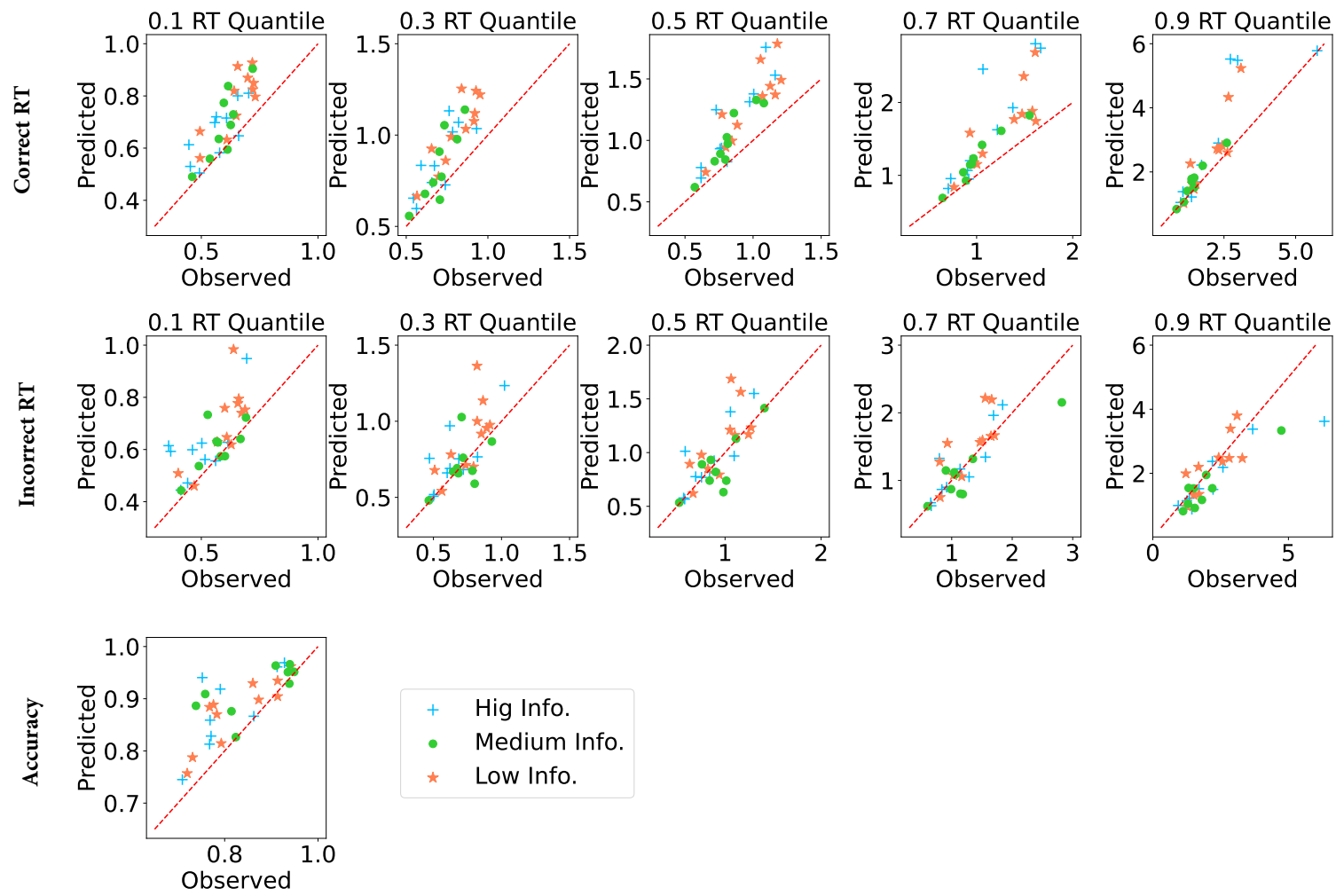

+ Hig Info.

- Medium Info.

* Low Info.

Figure A.2. Prediction of start-point variability model for different conditions. Top panel shows correct reaction time for in 10-th, 30-th, 50-th, 70-th, and 90-th quantile. Middle panel shows same quatiles for incorrect reaction times. Button panel illustrate the accuracy prediction. Each point shows one participant. 

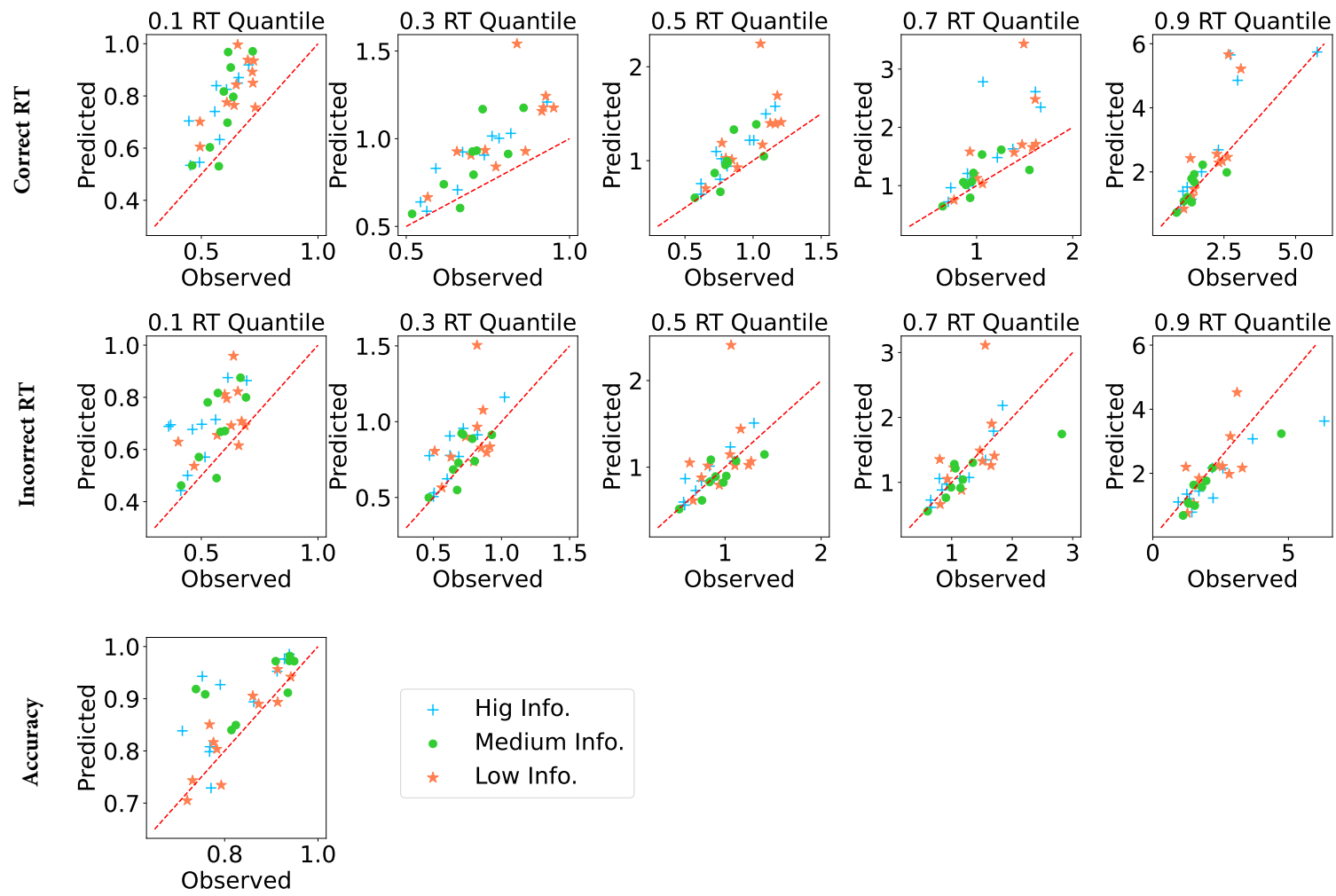

+ Hig Info.

- Medium Info.

* Low Info.

Figure A.3. Prediction of non-decision time variability model for different conditions. Top panel shows correct reaction time for in 10-th, 30-th, 50-th, 70-th, and 90-th quantile. Middle panel shows same quatiles for incorrect reaction times. Button panel illustrate the accuracy prediction. Each point shows one participant.

\section{Estimated decision threshold parameter of the models}

In this part the trends of the estimated decision threshold parameters $(a)$ are presented. Figure A.4 depicts the obtained decision threshold parameter $(a)$ for all four models. 

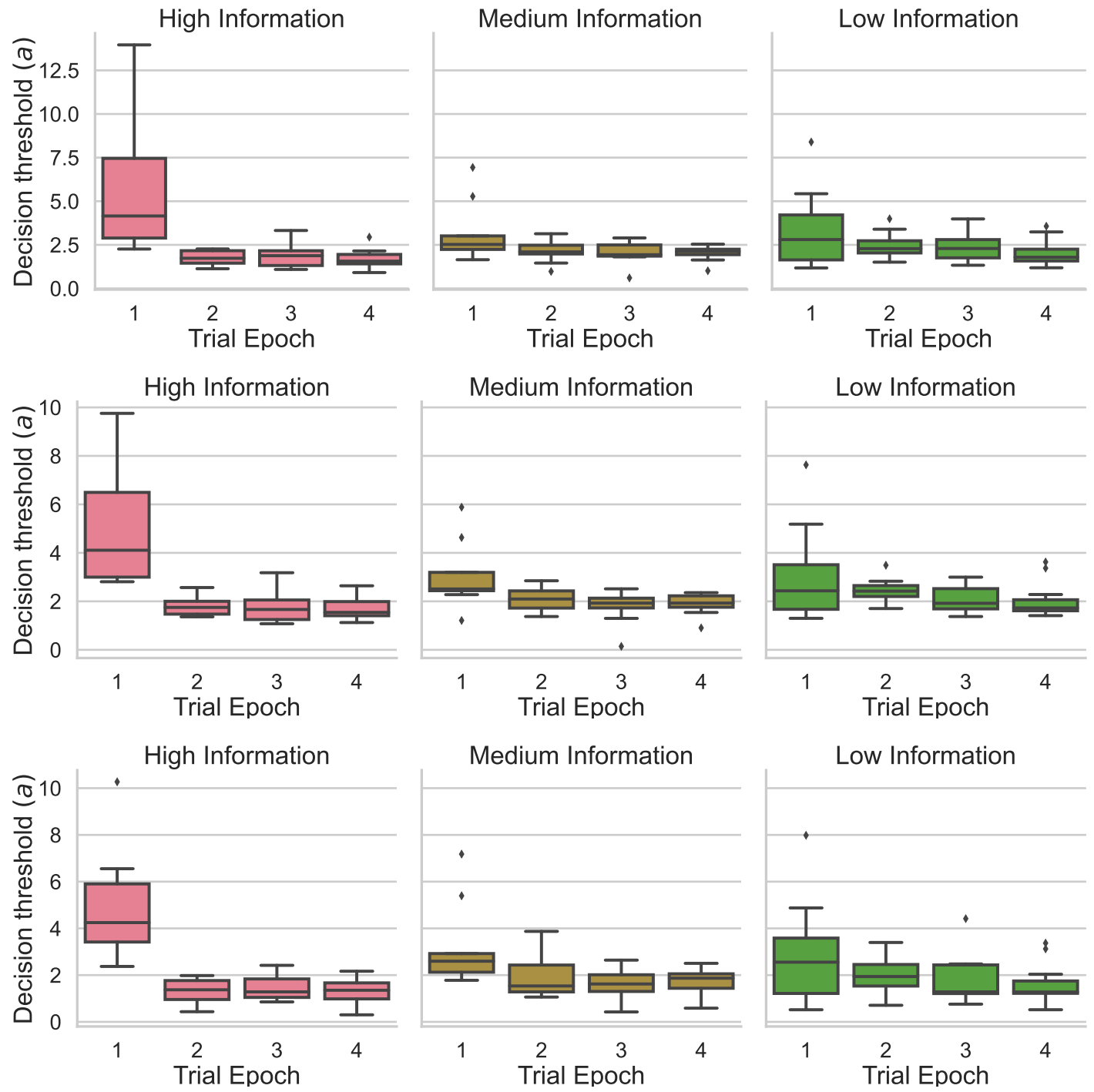

Figure A.4. Box plots depicting changes in the decision threshold parameter $(a)$, across trial epochs for the drift variability model (top panel), start-point variability model (middle panel), and nondecision time variability model (button panel).

\section{Estimated parameter from individual participant fits}

Estimated parameters from fitting all four models to individual participant data across trial epochs are shown in Figures A.5 to A.16. Each information condition is depicts in a separated figure. 


\section{$\alpha$ Varying Model}
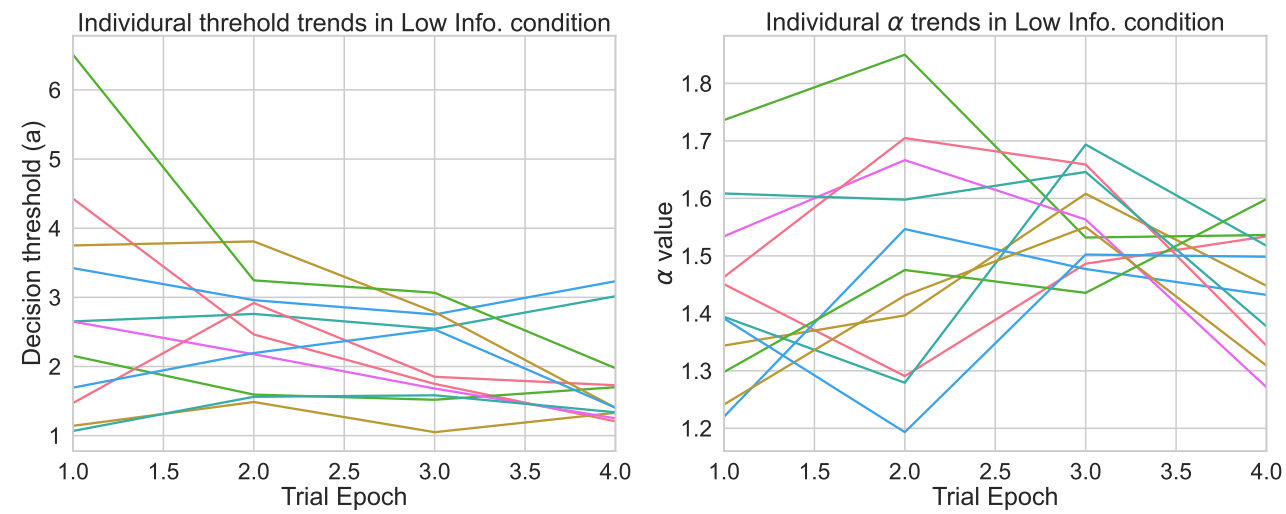

Figure A.5. Individual parameter estimates of the $\alpha$ varying model, for the participants in 'Low Information' condition for threshold (left panel) and $\alpha$ (right panel). Each line shows one participant. We refer the reader onto the online version of this article for the color version of this figure.
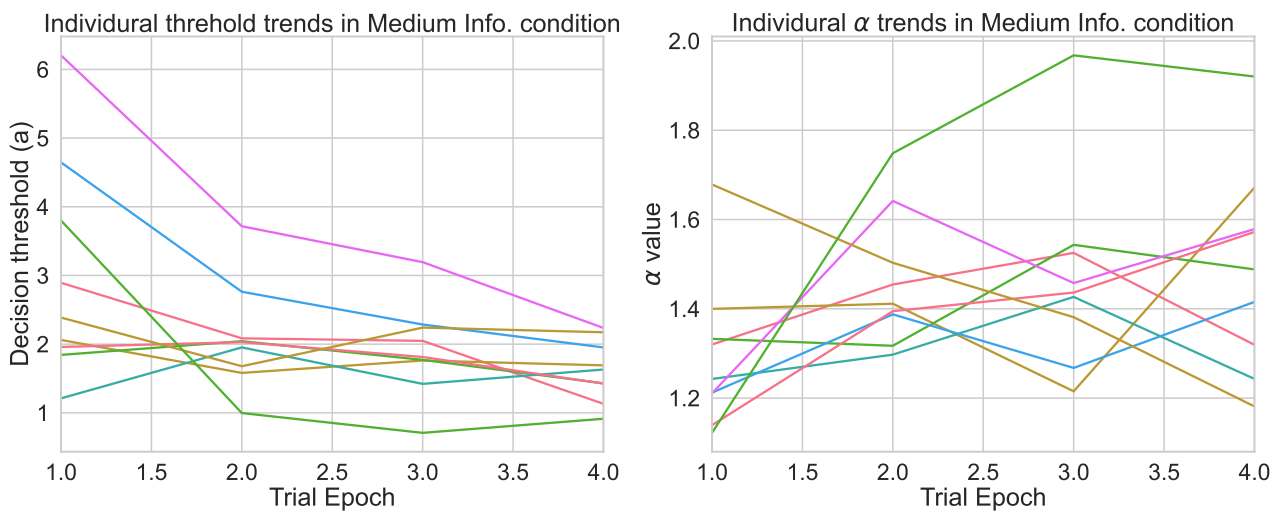

Figure A.6. Individual parameter estimates of the $\alpha$ varying model, for the participants in 'Medium Information' condition for threshold (left panel) and $\alpha$ (right panel). Each line shows one participant. We refer the reader onto the online version of this article for the color version of this figure. 

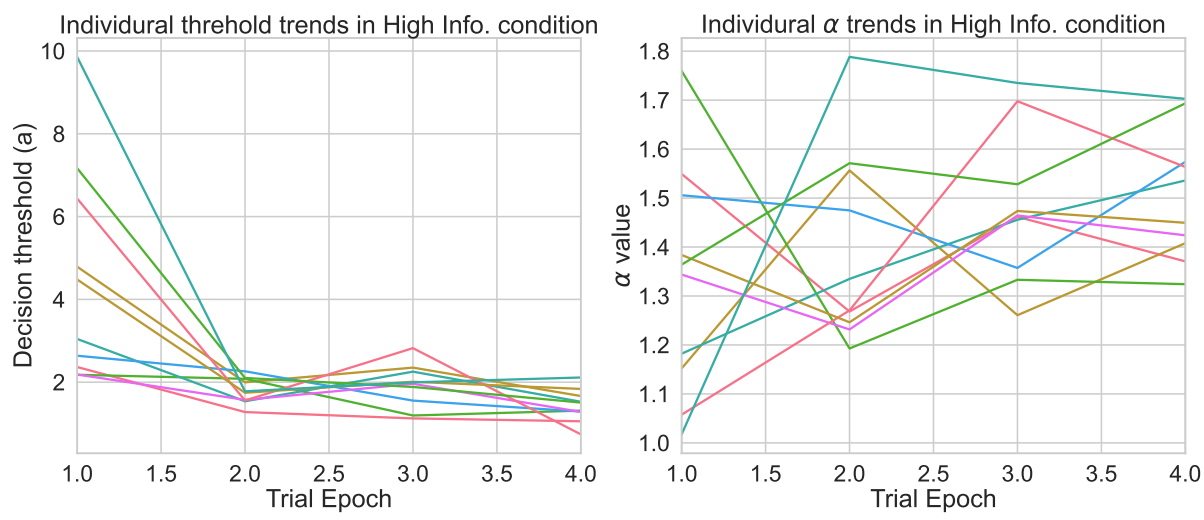

Figure A.7. Individual parameter estimates of the $\alpha$ varying model, for the participants in 'High Information' condition for threshold (left panel) and $\alpha$ (right panel). Each line shows one participant. We refer the reader onto the online version of this article for the color version of this figure.

\section{Drift Variability Model}
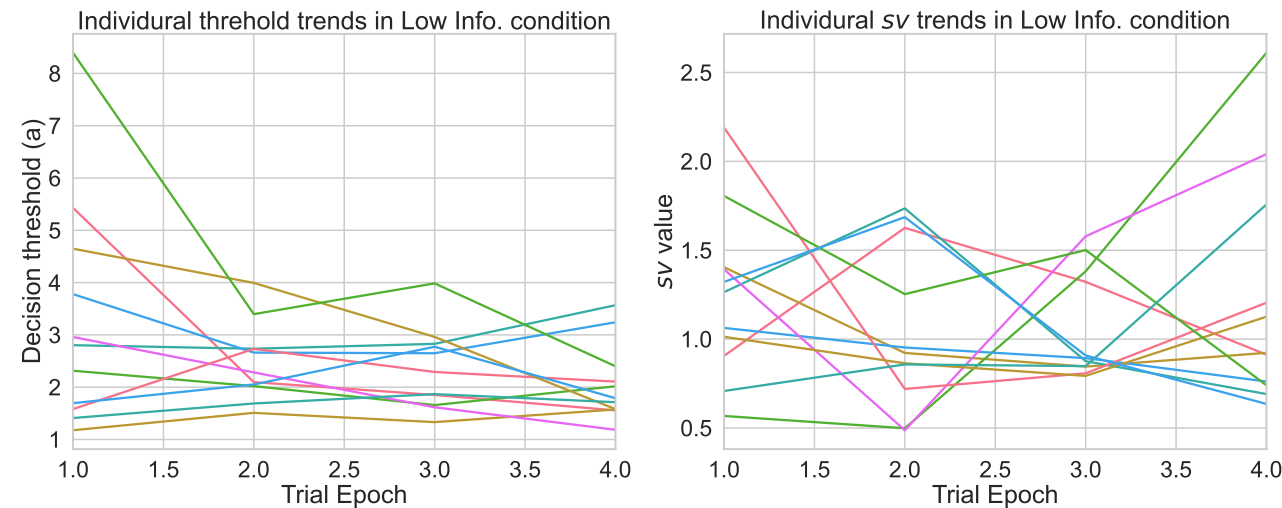

Figure A.8. Individual parameter estimates of the drift variability model, for the participants in 'Low Information' condition for threshold (left panel) and $s v$ (right panel). Each line shows one participant. We refer the reader onto the online version of this article for the color version of this figure. 

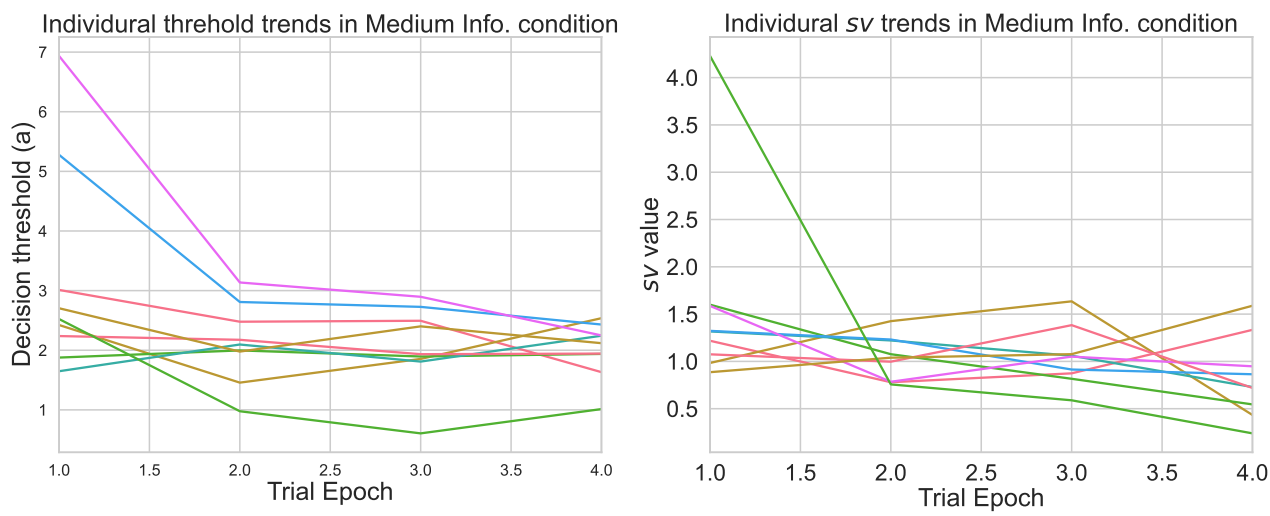

Figure A.9. Individual parameter estimates of the drift variability model, for the participants in 'Medium Information' condition for threshold (left panel) and sv (right panel). Each line shows one participant. We refer the reader onto the online version of this article for the color version of this figure.
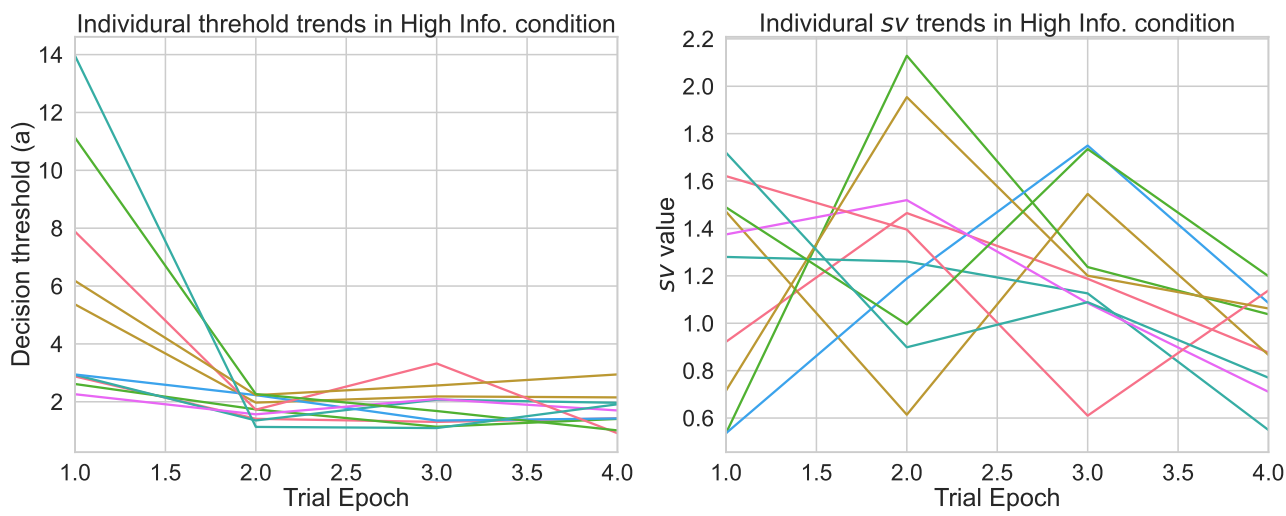

Figure A.10. Individual parameter estimates of the drift variability model, for the participants in 'High Information' condition for threshold (left panel) and $s v$ (right panel). Each line shows one participant. We refer the reader onto the online version of this article for the color version of this figure. 


\section{Start-Point Variability Model}
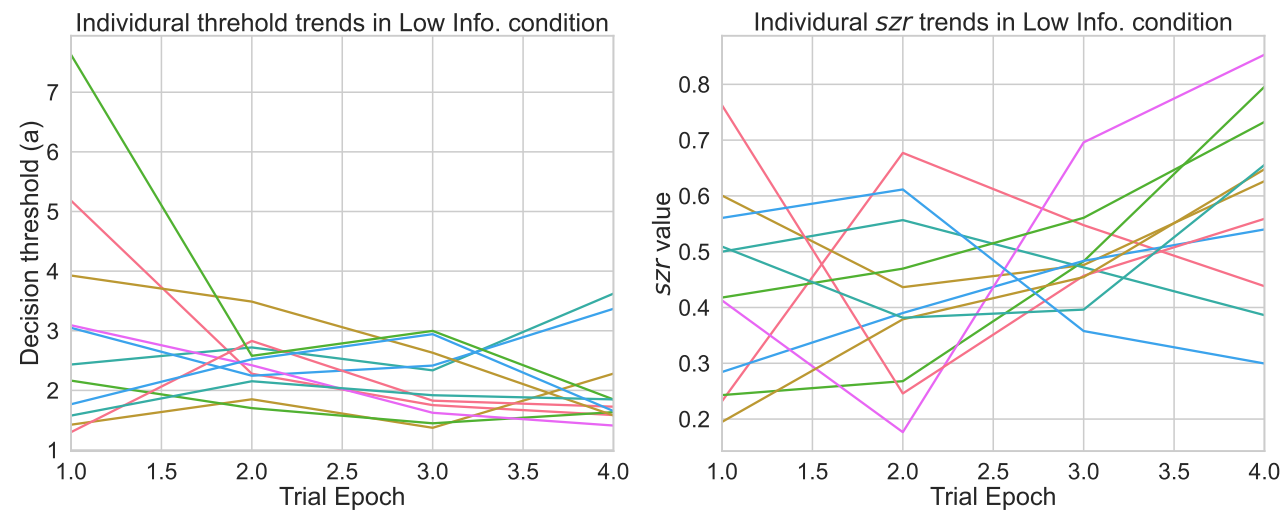

Figure A.11. Individual parameter estimates of the start-point variability model, for the participants in 'Low Information' condition for threshold (left panel) and $s z r$ (right panel). Each line shows one participant. We refer the reader onto the online version of this article for the color version of this figure.
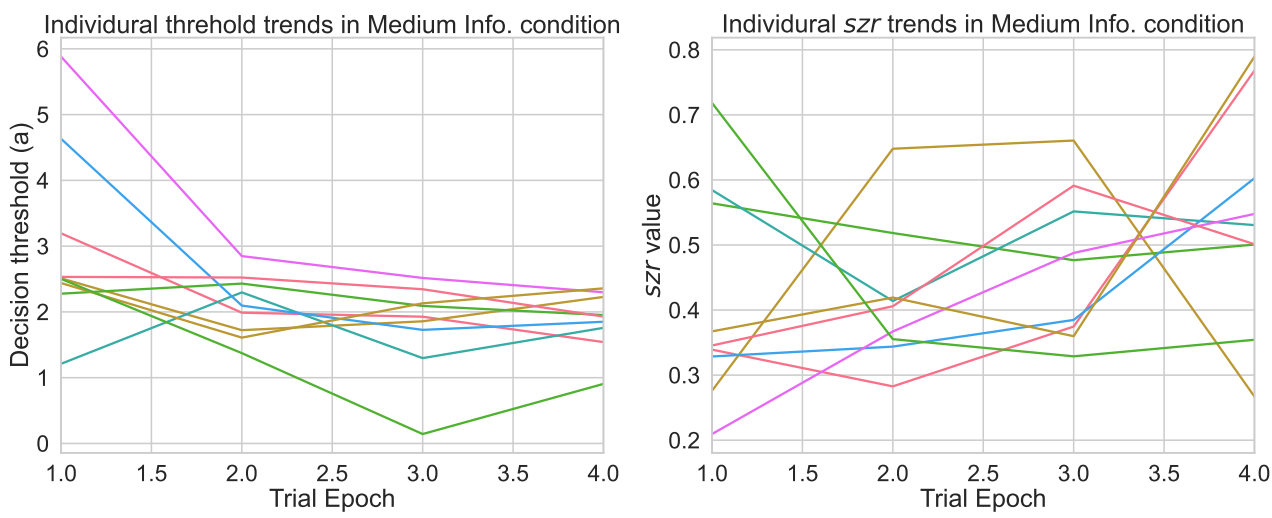

Figure A.12. Individual parameter estimates of the start-point variability model, for the participants in 'Medium Information' condition for threshold (left panel) and szr (right panel). Each line shows one participant. We refer the reader onto the online version of this article for the color version of this figure. 

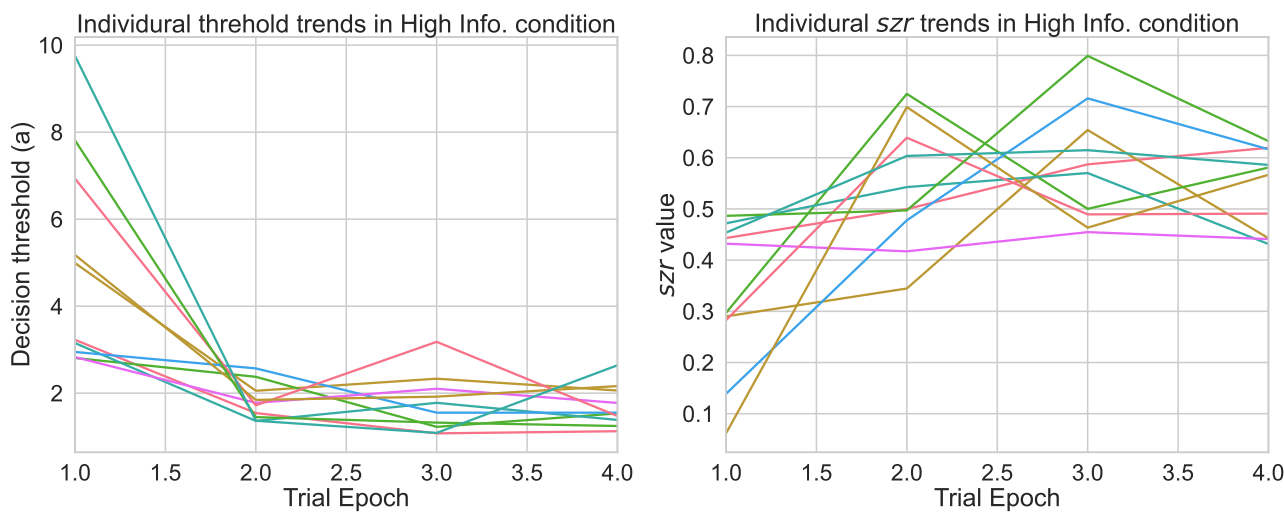

Figure A.13. Individual parameter estimates of the start-point variability model, for the participants in 'High Information' condition for threshold (left panel) and $s z r$ (right panel). Each line shows one participant. We refer the reader onto the online version of this article for the color version of this figure.

\section{Non-Decision Time Variability Model}
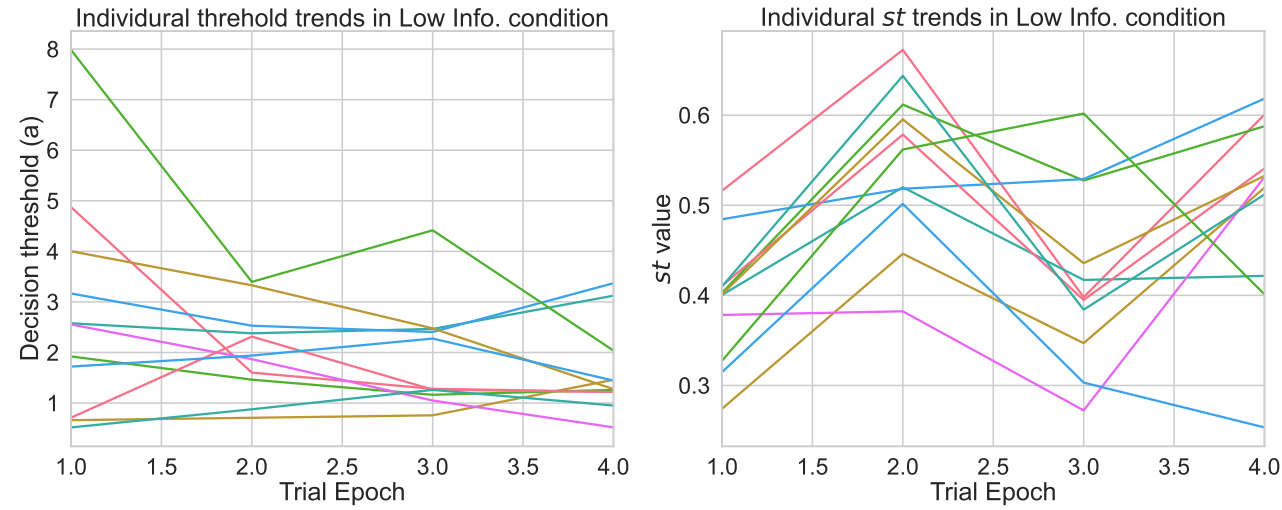

Figure A.14. Individual parameter estimates of the non-decision time variability model, for the participants in 'Low Information' condition for threshold (left panel) and st (right panel). Each line shows one participant. We refer the reader onto the online version of this article for the color version of this figure. 

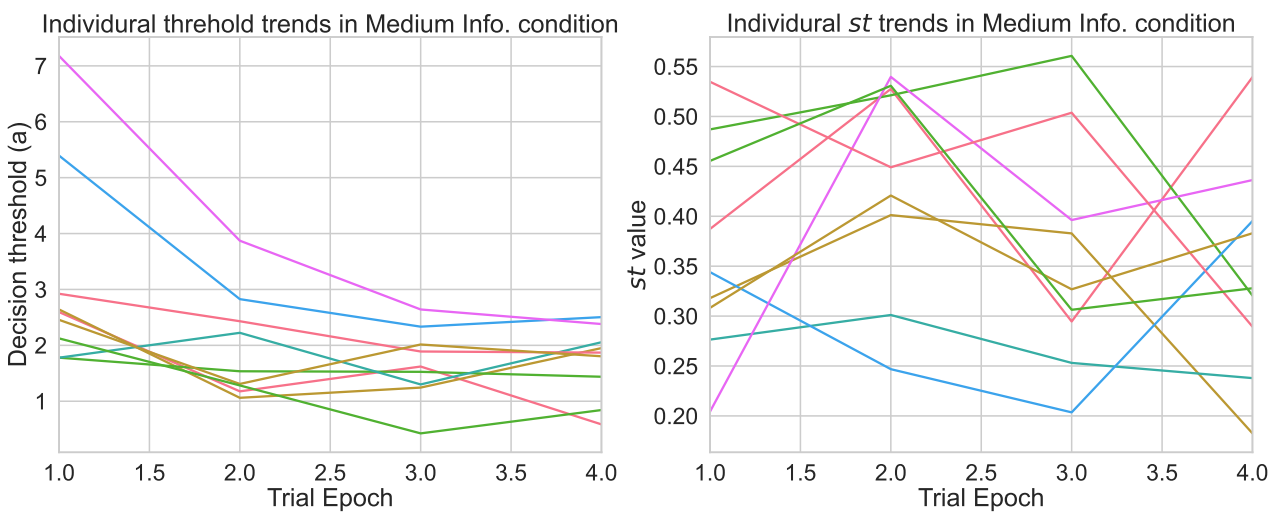

Figure A.15. The recovered parameters of the non-decision time variability model, for the participants in 'Medium Information' condition for threshold (left panel) and st (right panel). Each line shows one participant. We refer the reader onto the online version of this article for the color version of this figure.
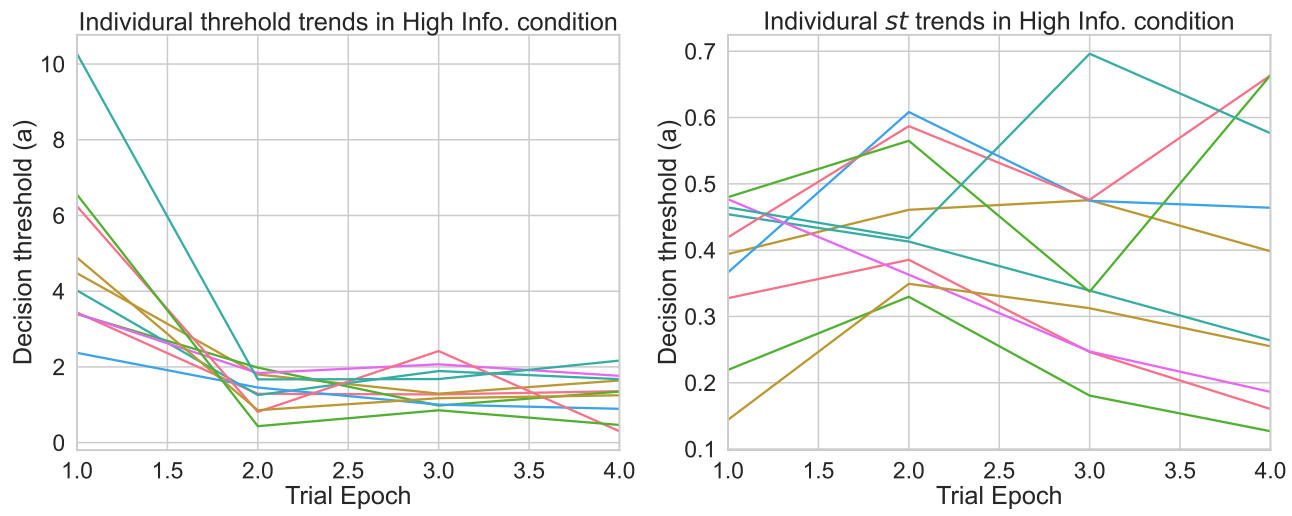

Figure A.16. The recovered parameters of the non-decision time variability model, for the participants in 'High Information' condition for threshold (left panel) and st (right panel). Each line shows one participant. We refer the reader onto the online version of this article for the color version of this figure. 